\title{
SUSY in the sky with gravitons
}

\author{
Gustav Uhre Jakobsen, ${ }^{a, b}$ Gustav Mogull, ${ }^{a, b}$ Jan Plefka ${ }^{a}$ and Jan Steinhoff ${ }^{b}$ \\ ${ }^{a}$ Institut für Physik und IRIS Adlershof, Humboldt-Universität zu Berlin, \\ Zum Großen Windkanal 2, D-12489 Berlin, Germany \\ ${ }^{b}$ Max-Planck-Institut für Gravitationsphysik (Albert-Einstein-Institut), \\ Mühlenberg 1, D-14476 Potsdam, Germany \\ E-mail: gustav.uhre.jakobsen@physik.hu-berlin.de, \\ gustav.mogull@aei.mpg.de, jan.plefka@hu-berlin.de, \\ jan.steinhoff@aei.mpg.de
}

ABSTRACT: Picture yourself in the wave zone of a gravitational scattering event of two massive, spinning compact bodies (black holes, neutron stars or stars). We show that this system of genuine astrophysical interest enjoys a hidden $\mathcal{N}=2$ supersymmetry, at least to the order of spin-squared (quadrupole) interactions in arbitrary $D$ spacetime dimensions. Using the $\mathcal{N}=2$ supersymmetric worldline action, augmented by finite-size corrections for the non-Kerr black hole case, we build a quadratic-in-spin extension to the worldline quantum field theory (WQFT) formalism introduced in our previous work, and calculate the two bodies' deflection and spin kick to sub-leading order in the post-Minkowskian expansion in Newton's constant $G$. For spins aligned to the normal vector of the scattering plane we also obtain the scattering angle. All $D$-dimensional observables are derived from an eikonal phase given as the free energy of the WQFT that is invariant under the $\mathcal{N}=2$ supersymmetry transformations.

KeYwords: Black Holes, Classical Theories of Gravity, Effective Field Theories, Extended Supersymmetry

ARXiv EPrint: 2109.04465 


\section{Contents}

1 Introduction 1

2 Supersymmetric worldline actions 4

2.1 Putting spin on the worldline 4

$2.2 \mathcal{N}=2$ spinning superparticle in a curved background 6

3 Comparison with a spinning compact body $\quad 8$

3.1 Traditional worldline action $\quad 8$

3.2 Identification with the $\mathcal{N}=2$ spinning particle $\quad 9$

$\begin{array}{lll}3.3 & \text { Approximate supersymmetry of the finite-size term } & 10\end{array}$

4 Spinning supersymmetric WQFT 11

$\begin{array}{lll}4.1 & \text { Feynman rules } & 13\end{array}$

$\begin{array}{lll}4.2 & \text { Recursive properties } & 15\end{array}$

$\begin{array}{ll}4.3 & \text { Observables from the eikonal phase } \\ & 16\end{array}$

$\begin{array}{lll}4.4 & \text { Background field symmetries } & 17\end{array}$

5 The eikonal phase and derived observables $\quad 19$

$\begin{array}{ll}5.12 \mathrm{PM} \text { eikonal phase } & 19\end{array}$

$\begin{array}{lll}5.2 & 1 \mathrm{PM} \text { observables } & 21\end{array}$

5.32 2PM observables 23

6 Conclusions 26

$\begin{array}{lr}\text { A Supersymmetry } & 28\end{array}$

$\begin{array}{lr}\text { B Integrals } & 29\end{array}$

\section{Introduction}

Our Universe is populated by massive astrophysical bodies (black holes, neutron stars and stars) that rotate intrinsically - they carry spin. Along their trajectories through space and time scattering events may occur that are mediated by the gravitational force. The two scattered bodies will be deflected, their spins will be altered and the scattering process will emit gravitational Bremsstrahlung, which could be detected in future-generation gravitational wave observatories on or near Earth. In this work we show that this astrophysical system enjoys a hidden, extended $(\mathcal{N}=2)$ supersymmetry that constrains the dynamics of the spinning scattering process - at least to the order of spin-squared (or quadrupole) 
interactions. The supersymmetry is manifested by anti-commuting worldline vectors $\psi^{a}$ attributed to the spin tensor of the body $\left(S^{a b} \sim \bar{\psi}^{[a} \psi^{b]}\right)$. These fermionic degrees of freedom are a natural ingredient in the recently developed worldline quantum field theory (WQFT) description of massive spinning bodies [1-3]. The appearance of supersymmetry in such a problem of genuine astrophysical interest was first realized in ref. [4] by studying hidden symmetries of the charged spinning black hole (Kerr-Newman) solution.

The investigation of classical gravitational scattering has a long history in general relativity [5-10], being naturally performed in a post-Minkowskian (PM) perturbative expansion in Newton's constant $G$ about a flat spacetime background $\left(g_{\mu \nu}=\eta_{\mu \nu}+\sqrt{32 \pi G} h_{\mu \nu}\right)$ with the graviton field $h_{\mu \nu}$. This unbound setup is different to the intensively studied postNewtonian (PN) expansion in both $G$ and relative velocity $\left(\frac{G m}{r} \sim v^{2}\right)$ often used for binary inspirals, i.e. massive bodies on bound orbits with separation $r$. The gravitational radiation emitted in the inspiral finally leads to a merger and is now routinely detected in gravitational wave observatories [11-14]. Even though Bremsstrahlung-emitting gravitational scattering events appear to be out of reach for current gravitational wave observatories due to their non-periodic signal and lower intensity [15-17] they represent interesting targets for future searches, calling for precision calculations.

It has also been emphasized recently that gravitational scattering is relevant for the study of binary inspirals. There exist various options for mapping between the bound and unbound problems, including reconstructing a gravitational two-body potential [18-22], or mapping directly between physical observables such as the scattering angle (unbound) and periastron advance (bound) [23, 24]. The scattering problem per se is the natural habitat for quantum field theory (QFT) that was largely designed to describe the scattering of elementary particles in collider experiments. Applying the classical limit of such a perturbative Feynman-diagrammatic expansion of the path integral of matter-coupled gravity in a PM expansion has proven to be highly efficient, and there are two popular QFT-based approaches.

The worldline effective field theory (EFT) formalism [25-27] models massive bodies as point-like particles moving along their worldlines coupled to the gravitational field. Spin effects are naturally incorporated by introducing a spin tensor and co-moving frame along the worldline [28-30]. Similarly, finite-size and tidal effects may be systematically included by coupling the worldline degrees of freedom to higher-dimensional operators dressed with Wilson coefficients (or Love numbers) [31]. Integrating out the graviton fluctuations in the path integral yields an effective action for the interaction of two spinning bodies - see refs. [28, 29] for reviews of the PN framework. Impressively high orders in the PN [32-37] and PM [38-41] expansions have also been established.

The second rapidly developing approach is the amplitudes-based formalism [19, 4247]. Massive elementary particles (scalars, spin- $1 / 2$ fermions, etc.) minimally coupled to the gravitational field are the avatars of black holes, neutron stars or stars; their $2 \rightarrow 2$ quantum scattering amplitudes are constructed by employing modern innovations such as generalized unitarity and the double copy, see [48-51] for reviews. Yet, the $2 \rightarrow 2$ amplitude is only the starting point for a subtle classical limit [52-54]. This procedure then yields the gravitational potential between two massive spinning bodies and observables such as the 
deflection and spin kick have been derived from it. A direct path from amplitudes to classical observables was introduced in ref. [52]. Nevertheless, the inclusion of spin poses certain challenges, in particular going beyond spin-squared interactions [55] due to the known hurdle of constructing an interacting higher-spin quantum field theory. Tidal and finitesize effects have also been included [56-58]. Amplitudes-based approaches can employ the powerful tools developed in collider physics and have led to impressively high-order calculations without spin $[44-46,54,59-61]$ and first results with spin $[55,62]$ including radiation effects [63-71]. A related variant of the amplitude approach is the heavy-particle EFT [7275], which enables a more straightforward classical limit of the amplitude from the outset.

The WQFT is a new formalism that unites these two approaches and clarifies their interrelations [1-3]. The worldline path integral representation of a massive particle's graviton-dressed propagator corresponds to the EFT worldline theory's path integral. Inserting this into the QFT S-matrix (represented as a time-ordered correlation function) a precise connection between the EFT and amplitudes-based approaches was given for the spinless case [1], which involves the classical $\hbar \rightarrow 0$ limit. A key feature of the WQFT approach - distinguishing it from the EFT approach — is that both the graviton field $h_{\mu \nu}$ and the worldline fluctuations about straight-line backgrounds (in the scattering case) are integrated out in the path integral. In short, the worldline degrees of freedom are also quantized. This leads to an economic Feynman-diagram-based perturbative solution to the equations of motion of the matter-graviton system.

In the WQFT, direct access to classical observables, such as the spinless particle deflection [1] and the time-domain asymptotic gravitational waveform $[2,3]$ emerging from the scattering encounter, has been made. The approach is economic in the sense that (a) one only computes the classically relevant contributions, circumventing the "super-classical" subtleties of the amplitudes-based approach, and (b) there is no need to determine a non-observable and gauge-dependent effective potential, thereafter solving the resulting equations of motion perturbatively as is done in the EFT approach. In fact, the WQFT formalism is to date the only approach used to successfully construct the asymptotic gravitational waveform of the QFT-based approaches with or without spin $[2,3]{ }^{1}$

In this paper we report on the inclusion of spin degrees of freedom in the WQFT. As mentioned above spin is introduced through anti-commuting worldline vectors, building upon refs. [4, 78-80]. In those works it was shown that the higher-spin $\mathcal{N} / 2$ field equations (in flat space) follow from quantizing an $\mathcal{N}$-extended supersymmetric particle augmenting the coordinate vector $x^{\mu}(\tau)$ by $\mathcal{N}$ anti-commuting vectors $\psi^{\mu}$. In (generic) curved spacetimes this is only possible up to $\mathcal{N}=2$ supersymmetry (or spin-1) as we will discuss in section 2. This limits our approach to the gravitational scattering of spinning objects up to interactions of quadratic order in spin (quadrupoles) at present. In our recent letter [3] we have already used this formalism to establish the asymptotic waveform of a spinning gravitational Bremsstrahlung event, extending the seminal work of Crowley, Kovacs and Thorne [5-7] to the spinning case.

\footnotetext{
${ }^{1}$ See refs. [47, 76, 77] for work on the gravitational Bremsstrahlung integrand or the related problem in electrodynamics in the amplitudes and EFT based approaches.
} 
Our spinning WQFT formalism innovates over existing approaches to classical spin based on corotating-frame variables $[28,29]$ in the effective field theory (EFT) of compact objects $[25-27,81,82]$ — see refs. [83-85] for the construction of PM integrands and refs. $[30,38]$ for the computation of worldline and spin deflections (shown to be in agreement with the amplitude based results [55, 62]). The EFT approach was applied to radiation also in the PN approximation [86-90] — see refs. [91, 92] for more traditional methods. Other approaches to PM spin effects can be found in refs. [72, 73, 93-102].

This paper is organized as follows. In section 2 we review the supersymmetric worldline formalism and give a detailed analysis of the $\mathcal{N}=2$ supersymmetric particle in a curved background. In section 3 we show that this theory is equivalent to the traditional description of spinning particles using spin and body-fixed frame fields, and explain how finite-size effects may be incorporated while maintaining the supersymmetry up to the desired quadratic-in-spin order. In section 4 we lay out the spinning WQFT and establish its Feynman rules, the relationship of the eikonal phase of a scattering event to the free energy of the spinning WQFT and show the hidden supersymmetry properties of the eikonal phase. Finally, in section 5 we compute a larger class of observables: the eikonal phase up to 2PM (next-to-leading) order from which we derive the deflection and spin kick. We close with concluding remarks and in the appendices give details of the $\mathcal{N}=2$ supersymmetry of the gauge-fixed spinning WQFT action and on the computation of the necessary integrals arising in section 5 .

\section{Supersymmetric worldline actions}

Extending the WQFT to include spin calls for a worldline theory of a relativistic spinning particle. In this section we review the first-order formulation of spinning particle actions where spin is represented by anti-commuting vector fields. Our main focus is the $\mathcal{N}=2$ supersymmetric theory in a generic curved background, which represents massive spinning bodies up to quadratic order in spin.

\subsection{Putting spin on the worldline}

To begin with recall the first-order formulation of the massless non-spinning particle in a gravitational background:

$$
\tilde{S}_{0}=-\int \mathrm{d} \tau\left[p_{M} \dot{x}^{M}-e H\right], \quad \text { with } \quad H=\frac{1}{2} p_{M} p_{N} g^{M N} .
$$

The transition to the massive case is easily done through a dimensional reduction setting $p^{M}=\left(p^{\mu}, m\right)$, which yields $H=\frac{1}{2}\left(p^{2}-m^{2}\right)$. We work with mostly minus signature and take the spacetime manifold to be $M^{1, D-1} \times S^{1}$. Eliminating the momentum through its algebraic equations of motion, $p^{\mu}=e^{-1} \dot{x}^{\mu}$, yields the second-order action

$$
S_{0}=-\int \mathrm{d} \tau\left[\frac{e^{-1}}{2} g_{\mu \nu} \dot{x}^{\mu} \dot{x}^{\nu}+\frac{e}{2} m^{2}\right],
$$

which is the "Polyakov" formulation of the non-spinning worldline theory enjoying a local reparametrization invariance $\tau \rightarrow \tau^{\prime}(\tau)$ under which $e$ transforms as $e \rightarrow e \dot{\tau}^{\prime}$. One may also 
eliminate the einbein $e$ using its algebraic equations of motion to arrive at the usual action for a massive particle $m \int \mathrm{d} s$. However, it is more convenient to choose the proper-time gauge $e=1 / m$ as this linearizes the graviton worldline interaction [1,38].

In order to include spin for the particle one adds real anti-commuting vector fields $\psi_{\alpha}^{a}(\tau)$ to the worldline degrees of freedom $[78,103,104]$. Here $\alpha=1, \ldots \mathcal{N}$ is a flavor index while $a=0, \ldots, D-1$ is the flat tangent space index related to the curved spacetime index $\mu=0, \ldots, D-1$ via the vielbein $e_{\mu}^{a}(x)$, with $g_{\mu \nu}=e_{\mu}^{a} e_{\nu}^{b} \eta_{a b}$ as usual. Of particular interest are the supersymmetric worldline theories which have been shown to describe the propagation of relativistic quantum fields of spin $1 / 2$ for $\mathcal{N}=1$ supersymmetry [103], spin 1 for $\mathcal{N}=2$ supersymmetry and generally spin $\mathcal{N} / 2$ for $\mathcal{N}$-extended supersymmetry [78, 104] in a flat spacetime background, respectively. The $\mathcal{N}$-extended supersymmetric spinning particle generalization of eq. $(2.1)$ in a flat $(D+1)$-dimensional spacetime background is ${ }^{2}$

$$
\tilde{S}_{N}=-\int \mathrm{d} \tau\left[p_{M} \dot{x}^{M}+\frac{i}{2} \psi_{\alpha}^{A} \dot{\psi}_{\alpha}^{B} \eta_{A B}-e H-i \chi^{\alpha} Q_{\alpha}-\frac{1}{2} f_{\alpha \beta} M^{\alpha \beta}\right]
$$

with the conserved charges

$$
H=\frac{1}{2} p^{2}, \quad Q_{\alpha}=p \cdot \psi_{\alpha}, \quad M_{\alpha \beta}=i \psi_{\alpha} \cdot \psi_{\beta} .
$$

The Lagrange multipliers of the einbein $e(\tau)$, the $\mathcal{N}$ gravitinos $\chi^{a}(\tau)$ and the $\mathrm{O}(\mathcal{N})$ gauge field $f_{\alpha \beta}(\tau)$ enforce the conservation of these charges. This gauged theory may be thought of as a locally supersymmetric worldline theory, i.e. $\mathcal{N}$-extended 1D supergravity, supersymmetrizing the reparametrization invariance of the spinless case eq. (2.1). Upon using the Poisson (resp. Dirac) brackets for the worldline fields

$$
\left\{x^{M}, p_{N}\right\}_{\text {P.B. }}=\delta_{N}^{M}, \quad\left\{\psi_{\alpha}^{A}, \psi_{\beta}^{B}\right\}_{\text {P.B. }}=-i \delta_{\alpha \beta} \eta^{A B},
$$

one derives the $\mathcal{N}$-extended supersymmetry algebra

$$
\begin{array}{rlrl}
\left\{Q_{\alpha}, Q_{\beta}\right\}_{\text {P.B. }} & =-2 i \delta_{\alpha \beta} H, & \left\{H, Q_{\beta}\right\}_{\text {P.B. }}=\left\{H, M_{\alpha \beta}\right\}_{\text {P.B. }}=0, \\
\left\{M_{\alpha \beta}, Q_{\gamma}\right\}_{\text {P.B. }}=-2 \delta_{\gamma[\alpha} Q_{\beta]}, & \left.\left\{M_{\alpha \beta}, M^{\gamma \delta}\right\}_{\text {P.B. }}=-4 \delta_{[\alpha}^{[\gamma} M_{\beta]}\right]^{[]}
\end{array}
$$

The charges $M_{\alpha \beta}$ generate an $\mathrm{O}(\mathcal{N}) R$-symmetry algebra. Again, a dimensional reduction of the theory from $D+1$ to $D$ dimensions, thereby setting $p^{M}=\left(p^{\mu}, m=\right.$ const $)$ and $\left(\psi^{A}=\right.$ $\left.\psi_{\alpha}^{a}, \theta_{\alpha}\right)$, yields the massive theory in $D$ dimensions - as in the spinless case of eq. (2.2).

The relevant question for our application is whether this theory may be embedded in an arbitrary curved spacetime background whilst preserving supersymmetry. This is only possible for $\mathcal{N} \leq 2$ describing a spin $0,1 / 2$ or spin 1 particle in a generic curved background [78-80, 104, 105]. For the $\mathcal{N}=4$ spinning particle a consistent quantization requires the backgound spacetime to be an Einstein manifold, i.e. $R_{\mu \nu}=\lambda g_{\mu \nu}$ [106]. As stated earlier, our focus is now the spinning $\mathcal{N}=2$ superparticle in a generic curved background, which allows us to describe spinning massive bodies up to quadratic order in spin.

\footnotetext{
${ }^{2}$ We take $M=(\mu, D)$ and $A=(a, D)$ with an $S^{1}$ in the $(D+1)$ th dimension.
} 


\section{$2.2 \mathcal{N}=2$ spinning superparticle in a curved background}

Following ref. [80] in order to prepare for the massive theory via dimensional reduction we consider the $\mathcal{N}=2$ spinning superparticle in the curved background $M^{1, D-1} \times S^{1}$. It is convenient to combine the two real Grassmann fields into one complex Grassmann worldline field via $\psi^{A}=\frac{1}{\sqrt{2}}\left(\psi_{1}^{A}+i \psi_{2}^{A}\right)$ and $\bar{\psi}^{A}=\frac{1}{\sqrt{2}}\left(\psi_{1}^{A}-i \psi_{2}^{A}\right)$. We note the Poisson (resp. Dirac) brackets

$$
\left\{x^{M}, p_{N}\right\}_{\text {P.B. }}=\delta_{N}^{M}, \quad\left\{\psi^{A}, \bar{\psi}^{B}\right\}_{\text {P.B. }}=-i \eta^{A B}
$$

The covariantization of the super-charges (2.4) takes the form

$$
Q=\psi^{a} e_{a}^{\mu}(x) \pi_{\mu}+\theta m, \quad \bar{Q}=\bar{\psi}^{a} e_{a}^{\mu}(x) \pi_{\mu}+\bar{\theta} m,
$$

where we have split off the fifth dimension explicitly $\psi^{5}:=\theta, p_{5}=m$ and introduced the covariantized four-momentum

$$
\pi_{\mu}:=p_{\mu}-i \omega_{\mu a b} \bar{\psi}^{a} \psi^{b},
$$

with the spin connection $\omega_{\mu}^{a b}=e_{\nu}^{a}\left(\partial_{\mu} e^{\nu b}+\Gamma_{\mu \lambda}^{\nu} e^{\lambda b}\right)$. The Hamiltonian may now be derived from the Poisson bracket $\{Q, \bar{Q}\}_{\text {P.B. }}$. Using $\left\{\pi_{\mu}, \pi_{\nu}\right\}_{\text {P.B. }}=i R_{\mu \nu a b} \bar{\psi}^{a} \psi^{b}$ and $\left\{\psi^{\mu}, \pi_{\nu}\right\}_{\text {P.B. }}=$ $\Gamma_{\nu \rho}^{\mu} \psi^{\rho}$ one finds

$$
\{Q, \bar{Q}\}_{\text {P.B. }}=-2 i[\underbrace{\frac{1}{2}\left(g^{\mu \nu} \pi_{\mu} \pi_{\nu}-m^{2}-R_{a b c d} \bar{\psi}^{a} \psi^{b} \bar{\psi}^{c} \psi^{d}\right)}_{H}]-2 \underbrace{\pi_{\mu} \bar{\psi}^{a} \psi^{b} T_{a b}^{\mu}}_{T} .
$$

Note that the last term $T$ couples to the torsion tensor $T_{\mu \rho}^{\nu}:=\Gamma_{[\mu \rho]}^{\nu}$, i.e. the antisymmetric part of the affine connection that vanishes in Einstein gravity. In addition $\{Q, Q\}_{\text {P.B. }}=0$ vanishes due to the cyclic identity for the Riemann tensor. Finally, in the complex basis for the Grassmann variables the internal $R$-symmetry turns into a U(1) symmetry generated by the charge

$$
J=\bar{\psi}^{a} \psi^{b} \eta_{a b}-\bar{\theta} \theta
$$

which obeys $\{J, Q\}_{\text {P.B. }}=-i Q$ and $\{J, \bar{Q}\}_{\text {P.B. }}=i \bar{Q}$.

In summary, the gauged first-order form of the $\mathcal{N}=2$ superparticle action in a curved spacetime background takes the form

$$
\begin{aligned}
\tilde{S}=-\int \mathrm{d} \tau & {\left[p_{\mu} \dot{x}^{\mu}+i \bar{\psi}^{a} \dot{\psi}^{b} \eta_{a b}-i \bar{\theta} \dot{\theta}-e H-i \bar{\chi} Q-i \chi \bar{Q}+a J\right] } \\
=-\int \mathrm{d} \tau & {\left[p_{\mu} \dot{x}^{\mu}+i \bar{\psi}^{a} \dot{\psi}^{b} \eta_{a b}-i \bar{\theta} \dot{\theta}-\frac{e}{2}\left(g^{\mu \nu} \pi_{\mu} \pi_{\nu}-m^{2}-R_{a b c d} \bar{\psi}^{a} \psi^{b} \bar{\psi}^{c} \psi^{d}\right)\right.} \\
& \left.\quad-i \pi_{\mu}\left(\bar{\chi} \psi^{\mu}-\bar{\psi}^{\mu} \chi\right)-i m(\bar{\chi} \theta-\bar{\theta} \chi)+a(\bar{\psi} \cdot \psi-\bar{\theta} \theta)\right]
\end{aligned}
$$


with the einbein $e$, the complex gravitino $\chi$ and the $\mathrm{U}(1)$ gauge field $a$. Eliminating $p_{\mu}$ by its algebraic equations of motion yields the action

$$
\begin{aligned}
S=-\int \mathrm{d} \tau[ & \frac{e^{-1}}{2} g_{\mu \nu}\left(\dot{x}^{\mu}-i \bar{\chi} \psi^{\mu}-i \chi \bar{\psi}^{\mu}\right)\left(\dot{x}^{\nu}-i \bar{\chi} \psi^{\nu}-i \chi \bar{\psi}^{\nu}\right) \\
& +i \bar{\psi}^{a}\left(\dot{\psi}_{a}+\dot{x}^{\mu} \omega_{\mu a b} \psi^{b}\right)+\frac{e}{2} R_{a b c d} \bar{\psi}^{a} \psi^{b} \bar{\psi}^{c} \psi^{d}+\frac{e}{2} m^{2}+a\left(\bar{\psi}^{a} \psi_{a}-\bar{\theta} \theta\right) \\
& \quad-i \bar{\theta} \dot{\theta}-i m(\bar{\chi} \theta+\chi \bar{\theta})] .
\end{aligned}
$$

A number of comments are now in order. Firstly, this theory is invariant under local reparametrization, supersymmetry and $\mathrm{U}(1)$ gauge symmetries under which both the worldline fields $x^{\mu}, \psi_{a}$ as well as $e, \chi, \bar{\chi}, a$ transform - see refs. [79, 80] for explicit formulae. Secondly, as shown in ref. [79], one may add a Chern-Simons term for the gauge field $a$ to the action

$$
S_{C S}=s \int \mathrm{d} \tau a, \quad s=\text { const }
$$

which is invariant under the $\mathrm{U}(1)$ transformation $\delta a=\dot{\alpha}, \delta \psi_{a}=i \alpha \psi_{a}$. Finally, one may set the Lagrange multipliers $\chi$ and $a$ to zero, yielding the constraints

$$
\begin{aligned}
\bar{\chi} & =0: & g_{\mu \nu} \psi^{\mu} \dot{x}^{\nu}+e m \theta & =0, \\
a & =0: & \eta_{a b} \bar{\psi}^{a} \psi^{b}-\bar{\theta} \theta & =s .
\end{aligned}
$$

We shall see in section 3 that these may be related to the spin-supplementary condition (SSC) as well as the conservation of the total spin in the traditional formulation of describing spinning compact objects.

The resulting second-order theory is then

$$
S^{\mathcal{N}=2}=-\int \mathrm{d} \tau\left[\frac{e^{-1}}{2} g_{\mu \nu} \dot{x}^{\mu} \dot{x}^{\nu}+i \bar{\psi}^{a}\left(\dot{\psi}_{a}+\dot{x}^{\mu} \omega_{\mu a b} \psi^{b}\right)+\frac{e}{2} R_{a b c d} \bar{\psi}^{a} \psi^{b} \bar{\psi}^{c} \psi^{d}+\frac{e}{2} m^{2}-i \bar{\theta} \dot{\theta}\right] .
$$

The fermionic extra dimensional contribution $\bar{\theta} \dot{\theta}$ is free and couples only to the other fields via the constraints $(2.15)$ - we drop it from now on. Moreover, it is convenient to further make the gauge choice $e=1 / m$ and rescale the fermions by $\sqrt{m}$ :

$$
\psi^{a} \rightarrow \sqrt{m} \psi^{a}, \quad \bar{\psi}^{a} \rightarrow \sqrt{m} \bar{\psi}^{a} .
$$

This renders the mass-shell constraint as

$$
\dot{x}^{\mu} \dot{x}_{\mu}=1+R_{a b c d} \bar{\psi}^{a} \psi^{b} \bar{\psi}^{c} \psi^{d}
$$

Therefore, strictly speaking $\tau$ is not the proper time in this gauge. This gauge fixed spinning worldline action (2.16) (setting $e=1 / m$ and rescaling the fermions) is invariant under the following global $\mathcal{N}=2$ supersymmetry transformations:

$$
\begin{aligned}
\delta x^{\mu} & =i e_{a}^{\mu}\left(\bar{\epsilon} \psi^{a}+\epsilon \bar{\psi}^{a}\right), \\
\delta \psi^{a} & =-\epsilon e_{\mu}^{a} \dot{x}^{\mu}-\delta x^{\mu} \omega_{\mu b}{ }^{a} \psi^{b}, \quad \delta \bar{\psi}^{a}=-\bar{\epsilon} e_{\mu}^{a} \dot{x}^{\mu}-\delta x^{\mu} \omega_{\mu}{ }^{a}{ }_{b} \bar{\psi}^{b},
\end{aligned}
$$


the analysis of which is relegated to appendix A. There is also a manifest global U(1) symmetry

$$
\delta_{a} \psi^{a}=i a \psi^{a}, \quad \delta_{a} \bar{\psi}^{a}=-i a \bar{\psi}^{a}, \quad \delta_{a} x^{\mu}=0,
$$

as well as the remnant of the reparametrization symmetry generated by $H$ of eq. (2.10), which is given by the commutator of two supersymmetries (2.19).

\section{Comparison with a spinning compact body}

In this section we demonstrate that the $\mathcal{N}=2$ spinning superparticle action (2.16) represents an alternative description of the classical physics of a spinning compact body moving in a gravitational background, up to and including terms quadratic in the spin (quadrupoles). For this we need to augment the spinning superparticle action by an additional term capturing finite-size effects and distinguishing black holes from (neutron) stars. Surprisingly, supersymmetry is preserved in an approximate sense under this extension of the theory and may be linked to the spin-supplementary condition (SSC).

\subsection{Traditional worldline action}

The traditional worldline action of a spinning compact body describes spin via a body-fixed frame $\Lambda_{A}{ }^{\mu}(\tau)$ along the worldline using the flat indices $A, B, \ldots$ that are distinct from the vielbein indices $a, b, \ldots$ from above. The first-order action takes the form $[81,82,107]$

$$
S^{\text {spin }}=-\int \mathrm{d} \tau[\pi_{\mu} \dot{x}^{\mu}+\frac{1}{2} S_{\mu \nu} \underbrace{\Lambda_{A}{ }^{\mu} \frac{D \Lambda^{A \nu}}{D \tau}}_{=: \Omega^{\mu \nu}}-\lambda\left(\pi_{\mu} \pi^{\mu}-\mathcal{M}^{2}\right)-\chi_{\mu} S^{\mu \nu}\left(\frac{\pi_{\nu}}{\sqrt{\pi^{2}}}+\Lambda_{0 \nu}\right)]
$$

Here $\frac{D}{D \tau}:=\dot{x}^{\mu} \nabla_{\mu}$ is the covariant derivative along the curve and the ortho-normal Lorentz body-fixed frame $\Lambda_{A}{ }^{\mu}$ satisfies $g_{\mu \nu} \Lambda_{A}{ }^{\mu} \Lambda_{B}{ }^{\nu}=\eta_{A B}$. Using $\Lambda_{A}{ }^{\mu}$ one may construct the (antisymmetric) angular velocity tensor $\Omega^{\mu \nu}$ as shown above. The Legendre dual of $\Omega^{\mu \nu}$ is the intrinsic angular momentum or spin tensor $S^{\mu \nu}$. The Lagrange multipliers $\lambda$ and $\chi_{\mu}$ enforce the mass-shell constraints $\pi^{2}=\mathcal{M}^{2}$ and the spin-supplementary condition (SSC) respectively; the latter arises from the necessity of constraining the antisymmetric $S^{\mu \nu}$ to carry only the physical rotational degrees of freedom of the compact body (i.e. three angles in 4d). If we gauge fix the time-like component of the body-fixed frame $\Lambda_{0}{ }^{\mu}=\pi^{\mu} / \sqrt{\pi^{2}}$ then the SSC takes the covariant form $S_{\mu \nu} \pi^{\nu}=0$. Instead, we find it more convenient to gauge fix via the choice $\chi_{\mu}=0$ for the Lagrange multiplier (analogous to fixing $e$ above) so we may drop the last term in the action, which approximately is equivalent to the covariant SSC $S_{\mu \nu} \pi^{\nu}=0+\mathcal{O}\left(S^{3}\right)$.

Starting at quadratic order in spins and linear order in curvature, the parity-invariant mass-shell constraint $\pi^{2}=\mathcal{M}^{2}$ receives curvature couplings [107]

$$
\mathcal{M}^{2}=m^{2}-\frac{1}{4} R_{\mu \nu \alpha \beta} S^{\mu \nu} S^{\alpha \beta}+C_{E} E_{\mu \nu} S^{\mu \rho} P_{\rho \sigma} S^{\nu \sigma}+\mathcal{O}\left(S^{3}\right)
$$


where $C_{E}$ is a Wilson coefficient induced by finite-size deformations (for a Kerr black hole $\left.C_{E}=0\right) .{ }^{3}$ We have introduced the "electric" curvature tensor $E_{\mu \nu}$ and projector $P_{\mu \nu}$ as

$$
\begin{aligned}
E_{\mu \nu} & =R_{\mu \alpha \nu \beta} \pi^{\alpha} \pi^{\beta} / m^{2} \\
P_{\mu \nu} & =g_{\mu \nu}-\pi_{\mu} \pi_{\nu} / \pi^{2}
\end{aligned}
$$

In four spacetime dimensions one may introduce the Pauli-Lubanski spin vector $S^{\mu}=$ $\frac{1}{2} \epsilon^{\mu \nu \alpha \beta} S_{\alpha \beta} \pi_{\nu} / m$ and the additional term takes the form $C_{E} E_{\mu \nu} S^{\mu} S^{\nu}$. Finally, $S^{\mu \nu} S_{\mu \nu}=$ $2 s^{2}$ is a constant along the worldline.

\subsection{Identification with the $\mathcal{N}=2$ spinning particle}

Identification of the traditional action for a spinning compact body eq. (3.1) with the $\mathcal{N}=2$ supersymmetric worldline theory of eq. (2.16) is made by identifying the spin tensor using

$$
S^{\mu \nu}=-2 i e_{a}^{\mu} e_{b}^{\nu} \bar{\psi}^{[a} \psi^{b]} .
$$

We have $S^{a b}=e_{\mu}^{a} e_{\nu}^{b} S^{\mu \nu}$ in a local Minkowski frame. So in a sense the worldline fermions $\psi^{a}$ are the quarks of the spin field $S^{a b}$. This identification is well-motivated by noting that the $\mathrm{SO}(1,3)$ Lorentz algebra:

$$
\left\{S^{a b}, S^{c d}\right\}_{\text {P.B. }}=\eta^{a c} S^{b d}+\eta^{b d} S^{a c}-\eta^{b c} S^{a d}-\eta^{a d} S^{b c},
$$

follows from $\left\{\psi^{a}, \bar{\psi}^{b}\right\}_{\text {P.B. }}=-i \eta^{a b}$ and provides the normalization in eq. (3.5). Note that the mass-shell constraint eq. (3.2) for $C_{E}=0$ directly maps with (3.5) to the Hamiltonian of the $\mathcal{N}=2$ theory (2.12), i.e. the Kerr black hole. Upon (seemingly) sacrificing supersymmetry the finite-size $C_{E}$ term may also be included by adding

$$
H_{E}:=m C_{E} E_{a b} \bar{\psi}^{a} \psi^{b} P_{c d} \bar{\psi}^{c} \psi^{d}
$$

to the spinning superparticle Hamiltonian $H$ in eq. (2.12). Using eq. (3.5) one can easily show that this agrees with the corresponding term in the spinning particle action (3.1). We will discuss the implications of this for SUSY at the end of this section.

How do we prove the equivalence of our $\mathcal{N}=2$ worldline SUSY theory (2.12), augmented by eq. (3.7), with the traditional formulation of eq. (3.1)? Taking inspiration from refs. [30, 81], we compare the Routhians $\mathcal{R}$ of the two theories. The Routhian to be considered is a Legendre transform of the Lagrangian with respect only to the spin degrees of freedom: it is a Hamiltonian with respect to the spin, but a Lagrangian with respect to the position of the particle. In the traditional spin formalism (3.1) switching to the Routhian conveniently eliminates all dependence on angular velocity tensor $\Omega^{\mu \nu}$ :

$$
\begin{aligned}
\mathcal{R}^{\text {spin }}: & =-\frac{1}{2} S_{a b} \Lambda_{A}^{a} \dot{\Lambda}^{A b}-L^{\text {spin }} \\
& =\pi_{\mu} \dot{x}^{\mu}-\frac{1}{2} \omega_{\mu, a b} \dot{x}^{\mu} S^{a b}+\lambda\left(\pi_{\mu} \pi^{\mu}-\mathcal{M}^{2}\right),
\end{aligned}
$$

\footnotetext{
${ }^{3}$ In the literature one often finds $C_{E} \rightarrow C_{E}-1$.
} 
where we used that $\Lambda_{A}^{a} \frac{D \Lambda^{A b}}{D \tau}=\Lambda_{A}^{a} \dot{\Lambda}^{A b}-\omega_{\mu}^{a b} \dot{x}^{\mu}$. Comparing this to the spinning superparticle action in eq. (2.12) augmented by the finite size term (3.7) the corresponding $\mathcal{N}=2$ SUSY Routhian takes the form

$$
\begin{aligned}
\mathcal{R}^{\mathcal{N}=2}:= & -i \eta_{a b} \bar{\psi}^{a} \dot{\psi}^{b}-L^{\mathcal{N}=2}-L^{E}=p_{\mu} \dot{x}^{\mu}+e\left(H+H_{E}\right) \\
= & \pi_{\mu} \dot{x}^{\mu}+i \omega_{\mu, a b} \dot{x}^{\mu} \bar{\psi}^{a} \psi^{b} \\
& -\frac{e}{2}\left(\pi_{\mu} \pi^{\mu}-m^{2}+R_{a b c d} \bar{\psi}^{a} \psi^{b} \bar{\psi}^{c} \psi^{d}+m C_{E} E_{a b} \bar{\psi}^{a} \psi^{b} P_{c d} \bar{\psi}^{c} \psi^{d}\right),
\end{aligned}
$$

having set $\theta=\bar{\theta}=0$ and used the definition of $\pi^{\mu}$ of eq. (2.9) in the last step. Upon identifying $\lambda=\frac{e}{2}$ we have a perfect match $\mathcal{R}^{\text {spin }}=\mathcal{R}^{\mathcal{N}=2}$, thereby also reproducing the finite-size term coupling to $C_{E}$ in $\mathcal{M}$ of $\mathcal{R}^{\text {spin }}$. We also note that the evolution of a generic function $F\left(\psi^{a}, \bar{\psi}^{a}\right)$ of the spinors is given by Hamilton's equation:

$$
\frac{\mathrm{d} F}{\mathrm{~d} \tau}=\{F, \mathcal{R}\}_{\text {P.B. }} .
$$

Choosing $F$ as $S^{\mu \nu}$ we see that $\mathcal{R}^{\text {spin }}=\mathcal{R}^{\mathcal{N}=2}$ guarantees consistency of the spin tensor's equation of motion between these two descriptions.

From our perspective, the Routhian has now served its purpose and we will not need it again. Our approach is therefore qualitatively different from other EFT-based methods, e.g. ref. [30], wherein one proceeds by solving Hamilton's equation (3.10) for $S^{\mu \nu}$. In that context, as we have discussed, the main benefit of the Routhian (3.8) over the Lagrangian (3.1) is that it does not depend on the angular velocity tensor $\Omega^{\mu \nu}$. However, by introducing the Grassmann vector $\psi^{a}$ at an early stage we have already gained this benefit. We will therefore continue to profit from our use of a fully Lagrangian-based formalism, using the SUSY action (2.12) augmented by the SUSY-breaking term (3.7), treating position and spin of the particles on an equal footing. This will allow us to perform calculations using a set of Feynman rules, to be derived in section 4 .

\subsection{Approximate supersymmetry of the finite-size term}

Finally, we discuss the implications of adding the SUSY-breaking finite-size term $H_{E}(3.7)$ to the Hamiltonian. In fact, this term does preserve SUSY approximately in the sense that the SUSY variation of it vanishes up to terms irrelevant for spin-squared interactions. This may be seen from the bracket of $H_{E}$ with the (undeformed) supercharge $Q$ : as $H_{E}$ is quartic in fermions the leading term in the bracket is cubic in fermions and reads

$$
\left\{Q, H_{E}\right\}_{\text {P.B. }}=m C_{E}(\underbrace{E_{\mu \nu} \pi^{\mu}}_{=0} e_{b}^{\nu} \psi^{b} P_{c d} \bar{\psi}^{c} \psi^{d}+E_{a b} \psi^{a} \psi^{b} \underbrace{\pi^{\gamma} P_{\gamma \rho}}_{=0} e_{d}^{\rho} \psi^{d})+\mathcal{O}\left(\psi^{5}\right),
$$

which vanishes by virtue of eqs. (3.3) and (3.4). Hence the full Hamiltonian $H+H_{E}$ is approximately supersymmetric. The SUSY-violating terms $\mathcal{O}\left(\psi^{5}\right)$ above will in turn receive corrections from the SUSY variation of putative spin-cubed additions to the Hamiltonian.

In fact, one may also incorporate $H_{E}$ into the SUSY algebra (2.6) in this approximate sense. Deforming the SUSY generators $Q$ and $\bar{Q}$ by $\mathcal{O}\left(\psi^{5}\right)$ terms via

$$
Q \rightarrow Q^{\prime}=Q+\frac{\psi \cdot \pi}{\pi^{2}} H_{E}, \quad \bar{Q} \rightarrow \bar{Q}^{\prime}=\bar{Q}+\frac{\bar{\psi} \cdot \pi}{\pi^{2}} H_{E}
$$


gives rise to the Poisson brackets

$$
\begin{aligned}
\left\{Q^{\prime}, \bar{Q}^{\prime}\right\}_{\text {P.B. }} & =-2 i H+\left\{Q, \frac{\bar{\psi} \cdot \pi}{\pi^{2}} H_{E}\right\}_{\text {P.B. }}+\left\{\bar{Q}, \frac{\psi \cdot \pi}{\pi^{2}} H_{E}\right\}_{\text {P.B. }}+\mathcal{O}\left(\psi^{6}\right) \\
& =-2 i\left(H+H_{E}\right)+\mathcal{O}\left(\psi^{6}\right)
\end{aligned}
$$

and $\left\{Q^{\prime}, Q^{\prime}\right\}_{\mathrm{PB}}=\mathcal{O}\left(\psi^{6}\right)$. Hence, we have an approximate closure of the $\mathcal{N}=2$ SUSY algebra. As our analysis is valid only up to spin-squared order we may neglect these higher-order fermionic terms and violations. This entails that the SUSY constraints alias SSC - hold only approximately for $C_{E} \neq 0$, i.e. we have $\pi \cdot \psi=\pi \cdot \bar{\psi}=0+\mathcal{O}\left(\psi^{5}\right)$ and $S_{\mu \nu} \pi^{\nu}=0+\mathcal{O}\left(S^{3}\right)$. In appendix A we discuss the Lagrangian version of this result in the gauge-fixed theory.

Finally, the U(1) $R$-symmetry is preserved by the finite-size extension. Having set the Lagrange multiplier $a$ to zero we also have the constraint $\bar{\psi} \cdot \psi=s$, which corresponds the conservation of spin length $S_{\mu \nu} S^{\mu \nu}=2 s^{2}$.

\section{Spinning supersymmetric WQFT}

In this section we use the $\mathcal{N}=2$ SUSY worldline action of section 2.2 to build a spinning generalization of the WQFT formalism [1], valid up to quadratic order in the spins. For each massive body $i$ we start from the worldline action (2.16):

$$
S^{(i)}=-m_{i} \int \mathrm{d} \tau\left[\frac{1}{2} g_{\mu \nu} \dot{x}_{i}^{\mu} \dot{x}_{i}^{\nu}+i \bar{\psi}_{i, a} \frac{D \psi_{i}^{a}}{D \tau}+\frac{1}{2} R_{a b c d} \bar{\psi}_{i}^{a} \psi_{i}^{b} \bar{\psi}_{i}^{c} \psi_{i}^{d}\right]
$$

where $\frac{D \psi_{i}^{a}}{D \tau}=\dot{\psi}_{i}^{a}+\dot{x}^{\mu} \omega_{\mu}^{a b} \psi_{i, b}$ and include the finite-size corrections (3.7)

$$
S_{E}^{(i)}:=-m_{i} C_{E, i} \int \mathrm{d} \tau R_{a \mu b \nu} \dot{x}_{i}^{\mu} \dot{x}_{i}^{\nu} \bar{\psi}_{i}^{a} \psi_{i}^{b} P_{c d} \bar{\psi}_{i}^{c} \psi_{i}^{d},
$$

with two distinct Wilson coefficients $C_{E, 1}$ and $C_{E, 2}$. Note that the projector reads $P_{a b}=$ $\eta_{a b}-e_{a \mu} e_{b \nu} \dot{x}^{\mu} \dot{x}^{\nu} / \dot{x}^{2}$ here. The two bodies interact via ordinary general relativity, appropriately described by the $D$-dimensional Einstein-Hilbert action and gauge-fixing term:

$$
S_{\mathrm{EH}}=-\frac{2}{\kappa^{2}} \int \mathrm{d}^{D} x \sqrt{-g} R, \quad S_{\mathrm{gf}}=\int \mathrm{d}^{D} x\left(\partial_{\nu} h^{\mu \nu}-\frac{1}{2} \partial^{\mu} h^{\nu}{ }_{\nu}\right)^{2},
$$

where $\kappa=\sqrt{32 \pi G}$ with Newton's constant $G$; the harmonic gauge-fixing term enforces $\partial_{\nu} h^{\mu \nu}=\frac{1}{2} \partial^{\mu} h_{\nu}^{\nu}$

In order to describe a scattering encounter we expand the worldline fields about their undeflected straight-line trajectories:

$$
x_{i}^{\mu}(\tau)=b_{i}^{\mu}+v_{i}^{\mu} \tau+z_{i}^{\mu}(\tau), \quad \psi_{i}^{a}(\tau)=\Psi_{i}^{a}+\psi_{i}^{\prime a}(\tau),
$$

with the new fields $z_{i}^{\mu}(\tau)$ and $\psi_{i}^{\prime a}(\tau)$ as perturbations. We also introduce the constant part of the spin tensor $S_{i}^{\mu \nu}(\tau)$ in the local frame:

$$
\mathcal{S}_{i}^{a b}=-2 i \bar{\Psi}_{i}^{[a} \Psi_{i}^{b]} .
$$


The bulk metric is expanded around a flat Minkowski vacuum:

$$
g_{\mu \nu}(x)=\eta_{\mu \nu}+\kappa h_{\mu \nu}(x),
$$

using a mostly minus metric signature $\eta_{\mu \nu}=\operatorname{diag}(+1,-1,-1,-1)$. The corresponding expansions of the vielbein $e_{\mu}^{a}$ and spin connection $\omega_{\mu}^{a b}$ are

$$
\begin{aligned}
e_{\mu}^{a} & =\eta^{a \nu}\left(\eta_{\mu \nu}+\frac{\kappa}{2} h_{\mu \nu}-\frac{\kappa^{2}}{8} h_{\mu \rho} h^{\rho}{ }_{\nu}+\mathcal{O}\left(\kappa^{3}\right)\right), \\
\omega_{\mu}{ }^{a b} & =-\kappa \partial^{[a} h^{b]}{ }_{\mu}-\frac{\kappa^{2}}{2} h^{\nu[a}\left(\partial^{b]} h_{\mu \nu}-\partial_{\nu} h^{b]}{ }_{\mu}+\frac{1}{2} \partial_{\mu} h^{b]}{ }_{\nu}\right)+\mathcal{O}\left(\kappa^{3}\right) .
\end{aligned}
$$

In this perturbative framework we no longer distinguish between curved $\mu, \nu, \ldots$ and tangent $a, b, \ldots$ indices. The background parameters $b_{i}^{\mu}, v_{i}^{\mu}$ and $\mathcal{S}_{i}^{\mu \nu}$, along with the masses of the two bodies $m_{i}$, constitute the physical data regarding the scattering in question. We may set $b \cdot v_{i}=0$, where $b^{\mu}=b_{2}^{\mu}-b_{1}^{\mu}$ is the relative impact parameter, and $v_{i} \cdot \Psi_{i}=v_{i} \cdot \bar{\Psi}_{i}=0$, implying $\mathcal{S}_{i}^{\mu \nu} v_{i \mu}=0$, without loss of generality - more on this in section 4.4.

In the WQFT framework $h_{\mu \nu}(x), z_{i}^{\mu}\left(\tau_{i}\right)$ and $\psi_{i}^{\prime \mu}\left(\tau_{i}\right)$ are promoted to quantum fields. The quantum theory is defined by the partition function:

$$
\begin{aligned}
\mathcal{Z}_{\mathrm{WQFT}}=e^{i \chi}:= & \text { const } \times \int D\left[h_{\mu \nu}\right] \int \prod_{i=1}^{2} D\left[z_{i}, \psi_{i}^{\prime}, \mathfrak{a}_{i}, \mathfrak{b}_{i}, \mathfrak{c}_{i}\right] \\
& \times \exp \left[i\left(S_{\mathrm{EH}}+S_{\mathrm{gf}}+\sum_{i=1}^{2}\left(S^{(i)}+S_{E}^{(i)}+S_{\text {ghost }}^{(i)}\right)\right)\right],
\end{aligned}
$$

where $\chi$ is the eikonal phase; the overall constant ensures that $\mathcal{Z}_{\mathrm{WQFT}}=1$ in the noninteracting case $(\kappa=0)$, so $\chi=0$. The additional terms $S_{\text {ghost }}^{(i)}$ arise from the need to write down a covariant path integral measure [108]:

$$
\begin{aligned}
\mathcal{D}[x] & =D[x] \prod_{0 \leq \sigma \leq T} \sqrt{-\operatorname{det} g_{\mu \nu}[x(\tau)]} \\
& =D[x] \int D[\mathfrak{a}, \mathfrak{b}, \mathfrak{c}] \exp [\underbrace{-i \int_{0}^{T} \mathrm{~d} \tau\left(\frac{1}{2} g_{\mu \nu}\left(\mathfrak{a}^{\mu} \mathfrak{a}^{\nu}+\mathfrak{b}^{\mu} \mathfrak{c}^{\nu}\right)\right)}_{i S_{\text {ghost }}}],
\end{aligned}
$$

which brings the "Lee-Yang" ghosts $\mathfrak{a}_{i}^{\mu}$ (commuting) and $\mathfrak{b}_{i}^{\mu}, \mathfrak{c}_{i}^{\mu}$ (anti-commuting) into the theory. On top, dimensional regularization of the path integral induces a finite counter term in terms of the Ricci scalar evaluated along the worldline trajectory $-\frac{1}{2} R[x(\tau)][108,109]$. However, both of these additions turn out to be irrelevant for the classical considerations in this work. Observables are calculated as expectation values in the WQFT:

$$
\begin{aligned}
\left\langle\mathcal{O}\left(h,\left\{x_{i}, \psi_{i}\right\}\right)\right\rangle:= & \mathcal{Z}_{\mathrm{WQFT}}^{-1} \int D\left[h_{\mu \nu}\right] \int \prod_{i=1}^{2} D\left[z_{i}, \psi_{i}^{\prime}, \mathfrak{a}_{i}, \mathfrak{b}_{i}, \mathfrak{c}_{i}\right] \mathcal{O}\left(h,\left\{x_{i}, \psi_{i}\right\}\right) \\
& \times \exp \left[i\left(S_{\mathrm{EH}}+S_{\mathrm{gf}}+\sum_{i=1}^{2}\left(S^{(i)}+S_{E}^{(i)}+S_{\text {ghost }}^{(i)}\right)\right)\right],
\end{aligned}
$$

To straightforwardly compute both these observables and the eikonal phase $\chi$ in practice we derive a set of momentum-space Feynman rules. These build on the non-spinning rules already derived in ref. [1], and have already been presented in ref. [3]. 


\subsection{Feynman rules}

As the Feynman rules originating from the Einstein-Hilbert action (4.3) are fairly conventional we do not dwell on them here; the graviton is simply re-expressed in momentum space as

$$
h_{\mu \nu}(x)=\int_{k} e^{i k \cdot x} h_{\mu \nu}(-k),
$$

where $\int_{k}:=\int \frac{\mathrm{d}^{D} k}{(2 \pi)^{D}}$ (the negative sign convention gives Feynman vertices with outgoing momenta). Spatial integration $\int \mathrm{d}^{D} x$ in eq. (4.3) gives rise to momentum-conserving delta functions at each $n$-point momentum-space graviton vertex; also including the gauge-fixing term $S_{\text {gf }}$ we read off the graviton propagator from the two-point function:

$$
\underset{\iota_{k}}{\mu \nu}=i \frac{P_{\mu \nu ; \rho \sigma}}{k^{2}+i \epsilon}, \quad P_{\mu \nu ; \rho \sigma}:=\eta_{\mu(\rho} \eta_{\sigma) \nu}-\frac{1}{D-2} \eta_{\mu \nu} \eta_{\rho \sigma} .
$$

The Feynman $i \epsilon$ prescription used here is consistent with purely conservative scattering, and will be sufficient at the $2 \mathrm{PM}$ order we shall be working at.

Next we consider the supersymmetric worldline action (4.1). The quadratic terms in $z_{i}^{\mu}$ and $\psi_{i}^{\prime \mu}$ are

$$
\left.S^{(i)}\right|_{\text {quadratic }}=-m_{i} \int \mathrm{d} \tau \eta_{\mu \nu}\left[\frac{1}{2} \dot{z}_{i}^{\mu} \dot{z}_{i}^{\nu}+i \bar{\psi}_{i}^{\prime \mu} \dot{\psi}_{i}^{\prime \nu}\right] .
$$

Writing the worldline fields in energy space,

$$
z_{i}^{\mu}(\tau)=\int_{\omega} e^{i \omega \tau} z_{i}^{\mu}(-\omega), \quad \psi_{i}^{\prime \mu}(\tau)=\int_{\omega} e^{i \omega \tau} \psi_{i}^{\prime \mu}(-\omega),
$$

where $\int_{\omega}:=\int \frac{\mathrm{d} \omega}{2 \pi}$, we read off the two worldline propagators:

$$
\begin{aligned}
& \underset{\omega}{\mu} \stackrel{\nu}{\bullet}=-i \frac{\eta^{\mu \nu}}{2 m_{i}}\left(\frac{1}{(\omega+i \epsilon)^{2}}+\frac{1}{(\omega-i \epsilon)^{2}}\right), \\
& \stackrel{\bullet_{\omega}}{\stackrel{\nu}{\bullet}}=-i \frac{\eta^{\mu \nu}}{2 m_{i}}\left(\frac{1}{\omega+i \epsilon}+\frac{1}{\omega-i \epsilon}\right) .
\end{aligned}
$$

As explained in ref. [1], the choice of $i \epsilon$ prescription determines the precise interpretation of the background parameters: with advanced or retarded prescriptions $b_{i, \pm \infty}^{\mu}, v_{i, \pm \infty}^{\mu}$ and $\Psi_{i, \pm \infty}^{\mu}$ are identified with the undeflected particle trajectories in the limit $\tau \rightarrow \pm \infty$. To leading order in $G$ the time-symmetric $i \epsilon$ prescription used here averages over these two possibilities, hence $\Psi_{i}^{\mu}=\frac{1}{2}\left(\Psi_{i,+\infty}^{\mu}+\Psi_{i,-\infty}^{\mu}\right)+\mathcal{O}\left(G^{2}\right), v_{i}^{\mu}=\frac{1}{2}\left(v_{i,+\infty}^{\mu}+v_{i,-\infty}^{\mu}\right)+\mathcal{O}\left(G^{2}\right)$ and $b_{i}^{\mu}=\frac{1}{2}\left(b_{i,+\infty}^{\mu}+b_{i,-\infty}^{\mu}\right)+\mathcal{O}\left(G^{2}\right)$.

Finally, we read off interaction vertices from the supersymmetric worldline action (4.1) and finite-size term (4.2). The worldline fields are written in energy space (4.14); as the graviton coupling to the worldline implicitly depends on $z_{i}^{\mu}$ we re-write it thus:

$$
\begin{aligned}
h_{\mu \nu}\left(x_{i}(\tau)\right) & =\int_{k} e^{i k \cdot\left(b_{i}+v_{i} \tau+z_{i}(\tau)\right)} h_{\mu \nu}(-k)=\sum_{n=0}^{\infty} \frac{i^{n}}{n !} \int_{k} e^{i k \cdot\left(b_{i}+v_{i} \tau\right)}\left(k \cdot z_{i}(\tau)\right)^{n} h_{\mu \nu}(-k) \\
& =\sum_{n=0}^{\infty} \frac{i^{n}}{n !} \int_{k, \omega_{1}, \ldots, \omega_{n}} e^{i k \cdot b_{i}} e^{i\left(k \cdot v_{i}+\sum_{j=1}^{n} \omega_{j}\right) \tau}\left(\prod_{j=1}^{n} k \cdot z_{i}\left(-\omega_{j}\right)\right) h_{\mu \nu}(-k) .
\end{aligned}
$$


Integration of the actions over proper time gives rise to energy-conserving delta functions $\delta(\omega):=2 \pi \delta(\omega)$, and we read off Feynman vertices from the interaction terms. The simplest vertex is the single-graviton source (suppressing the $i$ subscripts):

$$
\begin{aligned}
\sum_{\mu \nu}(k) & -i \frac{m \kappa}{2} e^{i k \cdot b} \delta(k \cdot v) \\
& \times\left(v^{\mu} v^{\nu}+i(k \cdot \mathcal{S})^{(\mu} v^{\nu)}-\frac{1}{2}(k \cdot \mathcal{S})^{\mu}(k \cdot \mathcal{S})^{\nu}+\frac{C_{E}}{2} v^{\mu} v^{\nu}(k \cdot \mathcal{S} \cdot \mathcal{S} \cdot k)\right),
\end{aligned}
$$

where $(k \cdot \mathcal{S})^{\mu}:=k_{\nu} \mathcal{S}^{\nu \mu}$, representing the linearized (in $h_{\mu \nu}$ ) stress-energy tensor. In the non-spinning case $\left(\mathcal{S}^{\mu \nu}=0\right)$ this precisely reproduces the corresponding vertex of ref. [1]; for a Kerr black hole $\left(C_{E}=0\right)$ the expression is consistent with an exponential representation of the linearized stress-energy tensor $[93,98,100]$ apparently valid to all orders in spin:

$$
\begin{aligned}
& h_{\mu \nu}(-k) T^{\mu \nu}(k)=m e^{i k \cdot b} \delta\left(k^{2}\right) \delta(k \cdot v)(v \cdot \epsilon)^{2} \exp \left(i \frac{k \cdot \mathcal{S} \cdot \epsilon}{v \cdot \epsilon}\right) \\
& \quad=m e^{i k \cdot b} \delta\left(k^{2}\right) \delta(k \cdot v) \epsilon_{\mu} \epsilon_{\nu}\left(v^{\mu} v^{\nu}+i(k \cdot \mathcal{S})^{(\mu} v^{\nu)}-\frac{1}{2}(k \cdot \mathcal{S})^{\mu}(k \cdot \mathcal{S})^{\nu}\right)+\mathcal{O}\left(\mathcal{S}^{3}\right),
\end{aligned}
$$

where the on-shell graviton is $h_{\mu \nu}(k)=\delta\left(k^{2}\right) \epsilon_{\mu} \epsilon_{\nu}$.

As the higher-point vertices become rapidly more complicated we provide only the ones required to compute the $2 \mathrm{PM}$ eikonal phase in section 4.3. Firstly, the graviton coupling to a single deflection mode is

$$
\begin{aligned}
& \sum_{h_{\mu \nu}(\omega)} z^{\rho}=\frac{m \kappa}{2} e^{i k \cdot b} \delta(k \cdot v+\omega) \\
\times & \left(2 \omega v^{(\mu} \delta_{\rho}^{\nu)}+v^{\mu} v^{\nu} k_{\rho}+i(k \cdot \mathcal{S})^{(\mu}\left(k_{\rho} v^{\nu)}+\omega \delta_{\rho}^{\nu)}\right)+\frac{1}{2} k_{\rho}(k \cdot \mathcal{S})^{\mu}(\mathcal{S} \cdot k)^{\nu}\right. \\
+ & \left.\frac{C_{E}}{2}\left(\left(2 \omega v^{(\mu} \delta_{\rho}^{\nu)}+v^{\mu} v^{\nu} k_{\rho}\right)(k \cdot \mathcal{S} \cdot \mathcal{S} \cdot k)-\omega^{2} k_{\rho}(\mathcal{S} \cdot \mathcal{S})^{\mu \nu}+2 \omega^{2}(k \cdot \mathcal{S} \cdot \mathcal{S})^{(\mu} \delta_{\rho}^{\nu)}\right)\right),
\end{aligned}
$$

which again reproduces the non-spinning case when $\mathcal{S}^{\mu \nu}=0$. The coupling to a single Grassmann-odd vector is

$$
\begin{gathered}
\sum_{h_{\mu \nu}(k)}=-i m \kappa e^{i k \cdot b} \delta(k \cdot v+\omega) \\
\quad \times\left(k_{[\rho} \delta_{\sigma]}^{(\mu}\left(v^{\nu)}-i(\mathcal{S} \cdot k)^{\nu)}\right)+i C_{E}\left(v^{(\mu} k_{\lambda}+\omega \delta_{\lambda}^{(\mu}\right)\left(v^{\nu)} k_{[\rho}+\omega \delta_{[\rho}^{\nu)}\right) \mathcal{S}_{\sigma]}^{\lambda}\right) \bar{\Psi}^{\sigma} .
\end{gathered}
$$

The vertex with an outgoing $\bar{\psi}^{\prime \rho}(\omega)$ line is the same, except with $\bar{\Psi}^{\sigma} \rightarrow \Psi^{\sigma}$. Finally, we 
require the two-graviton emission vertex from the worldline:

$$
\begin{aligned}
& r^{5^{5}} \frac{s^{3}}{\xi}=-\frac{m \kappa^{2}}{4} e^{i\left(k_{1}+k_{2}\right) \cdot b} \delta\left(\left(k_{1}+k_{2}\right) \cdot v\right) \\
& h_{\mu_{1} \nu_{1}}\left(k_{1}\right) h_{\mu_{2} \nu_{2}}\left(k_{2}\right) \\
& \times\left(\left(k_{1} \cdot \mathcal{S}\right)^{\mu_{2}} v^{\mu_{1}} \eta^{\nu_{1} \nu_{2}}-\mathcal{S}^{\mu_{1} \mu_{2}}\left(v^{\nu_{1}} k_{1}^{\nu_{2}}-\frac{1}{2} k_{1} \cdot v \eta^{\nu_{1} \nu_{2}}\right)\right. \\
& +i\left(\left(\mathcal{S} \cdot k_{1}\right)^{\mu_{1}}\left(\mathcal{S} \cdot k_{1}\right)^{\mu_{2}}+\frac{1}{2}\left(\mathcal{S} \cdot k_{2}\right)^{\mu_{1}}\left(\mathcal{S} \cdot k_{1}\right)^{\mu_{2}}-\frac{1}{2} \mathcal{S}^{\mu_{1} \mu_{2}}\left(k_{1} \cdot \mathcal{S} \cdot k_{2}\right)\right) \eta^{\nu_{1} \nu_{2}} \\
& +\frac{i}{4} k_{1} \cdot k_{2} \mathcal{S}^{\mu_{1} \nu_{2}} \mathcal{S}^{\mu_{2} \nu_{1}}-i k_{1}^{\nu_{2}}\left(\mathcal{S} \cdot\left(k_{1}+k_{2}\right)\right)^{\mu_{1}} \mathcal{S}^{\mu_{2} \nu_{1}} \\
& +i C_{E}\left(\left(2 k_{1} \cdot v\left(\mathcal{S} \cdot \mathcal{S} \cdot\left(k_{1}+k_{2}\right)\right)^{\mu_{2}} v^{\mu_{1}}-\frac{1}{2}\left(k_{1} \cdot v\right)^{2}(\mathcal{S} \cdot \mathcal{S})^{\mu_{1} \mu_{2}}\right.\right. \\
& \left.-\frac{1}{2}\left(k_{1} \cdot \mathcal{S} \cdot \mathcal{S} \cdot k_{2}\right) v^{\mu_{1}} v^{\mu_{2}}\right) \eta^{\nu_{1} \nu_{2}}-\frac{1}{2} k_{1} \cdot k_{2}(\mathcal{S} \cdot \mathcal{S})^{\nu_{1} \nu_{2}} v^{\mu_{1}} v^{\mu_{2}} \\
& +k_{1}^{\nu_{2}}\left(\mathcal{S} \cdot \mathcal{S} \cdot k_{2}\right)^{\nu_{1}} v^{\mu_{1}} v^{\mu_{2}}-k_{1}^{\nu_{2}}\left(\mathcal{S} \cdot \mathcal{S} \cdot k_{1}\right)^{\mu_{2}} v^{\mu_{1}} v^{\nu_{1}}-k_{1}^{\nu_{2}}\left(\mathcal{S} \cdot \mathcal{S} \cdot k_{2}\right)^{\mu_{2}} v^{\mu_{1}} v^{\nu_{1}} \\
& \left.\left.-(\mathcal{S} \cdot \mathcal{S})^{\mu_{2} \nu_{2}}\left(k_{1} \cdot v k_{2}^{\nu_{1}}-\frac{1}{2} k_{1} \cdot k_{2} v^{\nu_{1}}\right) v^{\mu_{1}}\right)\right)+(1 \leftrightarrow 2),
\end{aligned}
$$

which is implicitly symmetrized on $\left(\mu_{1}, \nu_{1}\right)$ and $\left(\mu_{2}, \nu_{2}\right)$.

\subsection{Recursive properties}

The Feynman rules (4.18), (4.20) and (4.21) satisfy recursive properties:

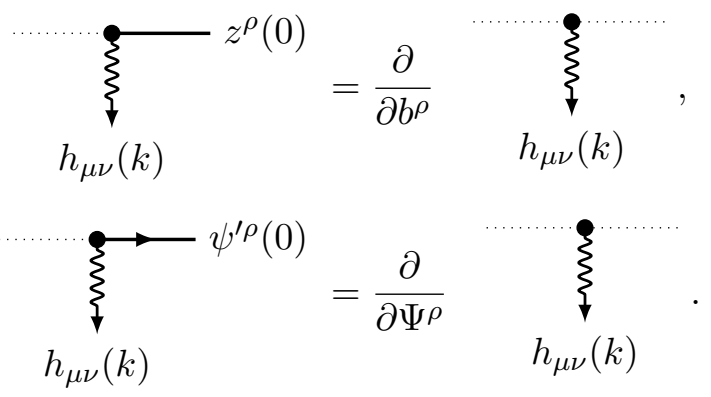

In ref. [1] (the non-spinning case) the first relationship was generalized to $n$ points:

$$
\sum_{z^{\rho^{\prime}}\left(\omega_{1}\right)}^{z^{\rho_{n}}\left(\omega_{n}\right)}=\frac{\partial}{\partial b^{\rho_{n+1}}}
$$

In words: a vertex with $(n+1)$ external $z^{\mu}$ particles, and $\omega_{n+1}=0$, is given by a derivative with respect to the impact parameter $b^{\mu}$ of the corresponding $n$-point vertex. We claim this continues to hold when spin is included, and that eq. (4.23b) generalizes similarly, 
regardless of what other external lines are present on the vertex. In the non-spinning case we confirmed this recursive property using an analytic expression for the worldline vertices:

$$
\begin{aligned}
& \sum^{z^{\rho_{n}}\left(\omega_{n}\right)} z^{\rho_{1}}\left(\omega_{1}\right)=i^{n-1} m \kappa e^{i k \cdot b} \delta\left(k \cdot v+\sum_{i=1}^{n} \omega_{i}\right) \\
& \times\left(\frac{1}{2}\left(\prod_{i=1}^{n} k_{\rho_{i}}\right) v^{\mu} v^{\nu}+\sum_{i=1}^{n} \omega_{i}\left(\prod_{j \neq i}^{n} k_{\rho_{j}}\right) v^{(\mu} \delta_{\rho_{i}}^{\nu)}+\sum_{i<j}^{n} \omega_{i} \omega_{j}\left(\prod_{l \neq i, j}^{n} k_{\rho_{l}}\right) \delta_{\rho_{i}}^{(\mu} \delta_{\rho_{j}}^{\nu)}\right) .
\end{aligned}
$$

With the inclusion of spin, however, we no longer have such a compact expression and therefore argue differently.

At the Lagrangian level these properties follow straightforwardly from

$$
\frac{\partial L^{(i)}(\tau)}{\partial b_{i}^{\mu}}=\frac{\partial L^{(i)}(\tau)}{\partial z_{i}^{\mu}(\tau)}, \quad \frac{\partial L^{(i)}(\tau)}{\partial \Psi_{i}^{\mu}}=\frac{\partial L^{(i)}(\tau)}{\partial \psi_{i}^{\prime \mu}(\tau)}
$$

where $S^{(i)}+S_{E}^{(i)}=\int \mathrm{d} \tau L^{(i)}(\tau)$ (we are now ignoring the ghosts). The former is true simply because the Lagrangian $L^{(i)}$ depends on $b_{i}^{\mu}$ and $z_{i}^{\mu}$ only implicitly via $x_{i}^{\mu}(\tau)=$ $b_{i}^{\mu}+\tau v_{i}^{\mu}+z_{i}^{\mu}(\tau)$; the latter because $L^{(i)}$ depends on spin only via $\psi_{i}^{\mu}(\tau)=\Psi_{i}^{\mu}+\psi_{i}^{\prime \mu}(\tau)$ (and its complex conjugate). In energy space the action therefore generically depends on $x_{i}^{\mu}\left(\omega_{j}\right)=\delta\left(\omega_{j}\right) b_{i}^{\mu}-i \delta^{\prime}\left(\omega_{j}\right) v_{i}^{\mu}+z_{i}^{\mu}\left(\omega_{j}\right)$ and $\psi_{i}^{\mu}\left(\omega_{j}\right)=\delta\left(\omega_{j}\right) \Psi_{i}^{\mu}+\psi_{i}^{\prime \mu}\left(\omega_{j}\right)$ for the collection of energies $\left\{\omega_{j}\right\}$. So, in this case a derivative with respect to the background parameter $b_{i}^{\mu}$ or $\Psi_{i}^{\mu}$ is equivalent to one with respect to the corresponding perturbation $z_{i}^{\mu}\left(\omega_{j}\right)$ or $\psi_{i}^{\prime \mu}\left(\omega_{j}\right)$, if we set $\omega_{j}=0$ (as implied by the delta function). In the next section we use these properties to obtain observables from the eikonal phase $\chi$.

\subsection{Observables from the eikonal phase}

The eikonal phase $\chi$ is a scalar with a privileged role in the WQFT, containing knowledge of both the classical impulse $\left\langle\Delta p_{i, \mu}\right\rangle$ and spin kick $\left\langle\Delta S_{i, \mu \nu}\right\rangle$. To recover these observables we use the recursive properties of the worldline vertices (4.26); from the action $S=S_{\mathrm{EH}}+$ $S_{\mathrm{gf}}+\sum_{i=1}^{2} \int \mathrm{d} \tau L^{(i)}(\tau)$ these may be re-expressed as

$$
\begin{aligned}
\frac{\partial S}{\partial b_{i}^{\mu}} & =\int_{-\infty}^{\infty} \mathrm{d} \tau\left(\frac{\partial L^{(i)}}{\partial x_{i}^{\mu}(\tau)}-\frac{\mathrm{d}}{\mathrm{d} \tau} \frac{\partial L^{(i)}}{\partial \dot{x}_{i}^{\mu}(\tau)}\right)-\underbrace{\left[p_{i, \mu}\right]_{\tau=-\infty}^{\tau=\infty}}_{\Delta p_{i, \mu}}, \\
\frac{\partial S}{\partial \Psi_{i}^{\mu}} & =\int_{-\infty}^{\infty} \mathrm{d} \tau\left(\frac{\partial L^{(i)}}{\partial \psi_{i}^{\mu}(\tau)}-\frac{\mathrm{d}}{\mathrm{d} \tau} \frac{\partial L^{(i)}}{\partial \dot{\psi}_{i}^{\mu}(\tau)}\right)+i m_{i} \underbrace{\left[\bar{\psi}_{i, \mu}\right]_{\tau=-\infty}^{\tau=\infty}}_{\Delta \bar{\psi}_{i, \mu}},
\end{aligned}
$$

where $p_{i, \mu}=-\frac{\partial L^{(i)}}{\partial \dot{x}_{i}^{\mu}}$ and $i m_{i} \bar{\psi}_{i, \mu}=\frac{\partial L^{(i)}}{\partial \dot{\psi}_{i}^{\mu}}$. As the first terms define the classical (EulerLagrange) equations of motion for $x_{i}^{\mu}$ and $\psi_{i}^{\mu}$, their expectation values in the WQFT vanish. Therefore, by taking derivatives of the free energy $-i \log \mathcal{Z}_{\mathrm{WQFT}}=\chi$ of eq. (4.8) 
with respect to $b_{i}^{\mu}, \Psi_{i}^{\mu}$ and $\bar{\Psi}_{i}^{\mu}$ and using eq. (4.27) we see that ${ }^{4}$

$$
\begin{aligned}
\left\langle\Delta p_{i, \mu}\right\rangle & =-\frac{\partial \chi}{\partial b_{i}^{\mu}}, \\
i m_{i}\left\langle\Delta \psi_{i, \mu}\right\rangle & =\frac{\partial \chi}{\partial \bar{\Psi}_{i}^{\mu}}=-2 i \Psi_{i}^{\nu} \frac{\partial \chi}{\partial \mathcal{S}_{i}^{\mu \nu}}, \\
i m_{i}\left\langle\Delta \bar{\psi}_{i, \mu}\right\rangle & =\frac{\partial \chi}{\partial \Psi_{i}^{\mu}}=-2 i \bar{\Psi}_{i}^{\nu} \frac{\partial \chi}{\partial \mathcal{S}_{i}^{\mu \nu}} .
\end{aligned}
$$

We have used the fact that $\chi$ depends on $\Psi_{i}^{\mu}$ and $\bar{\Psi}_{i}^{\mu}$ only implicitly via $\mathcal{S}_{i}^{\mu \nu}=-2 i \bar{\Psi}_{i}^{[\mu} \Psi_{i}^{\nu]}$. The expectation value of the spin kick $\Delta S_{i}^{\mu \nu}$ is therefore recovered as

$$
\begin{aligned}
\left\langle\Delta S_{i}^{\mu \nu}\right\rangle & =-2 i \bar{\Psi}_{i}^{[\mu}\left\langle\Delta \psi_{i}^{\nu]}\right\rangle-2 i\left\langle\Delta \bar{\psi}_{i}^{[\mu}\right\rangle \Psi_{i}^{\nu]} \\
& =\frac{4}{m_{i}} \mathcal{S}_{i}^{\rho[\mu} \frac{\partial \chi}{\partial \mathcal{S}_{i, \nu]}{ }^{\rho}} .
\end{aligned}
$$

For any field perturbed around a background expectation value, the expected "kick" of that field is therefore extracted from the eikonal phase by taking a derivative with respect to the corresponding background parameter.

In the special case of aligned spins to the scattering plane we can also determine the scattering angle $\theta$, given in terms of the momentum impulse as

$$
\sin \left(\frac{\theta}{2}\right)=\frac{\left|\Delta p_{i}\right|}{2 p_{\infty}}, \quad \quad p_{\infty}=\frac{m_{1} m_{2}}{E} \sqrt{\gamma^{2}-1}
$$

where $\left|\Delta p_{i}\right|:=\sqrt{-\left\langle\Delta p_{i}\right\rangle^{2}}, p_{\infty}$ is the center-of-mass momentum, the total energy is $E=$ $\sqrt{m_{1}^{2}+m_{2}^{2}+2 \gamma m_{1} m_{2}}$ and $\gamma=v_{1} \cdot v_{2}$. From the eikonal phase the scattering angle is directly recovered as

$$
\sin \left(\frac{\theta}{2}\right)=-\frac{1}{2 p_{\infty}} \frac{\partial \chi}{\partial|b|} .
$$

Using these relations, in sections 5.2 and 5.3 we will calculate the momentum impulse, spin kick and aligned-spin scattering angle at $1 \mathrm{PM}$ and $2 \mathrm{PM}$ order respectively.

\subsection{Background field symmetries}

Invariance of the action under the SUSY transformations (2.19) (see appendix A for details), the U(1) symmetry (2.20) and translation invariance along the worldline has physical consequences for these observables derived from the eikonal phase. After integrating out the worldline fluctuations $z_{i}^{\mu}$ and $\psi_{i}^{\prime \mu}$, for each transformation there is a flat-space remnant of these symmetries on the background parameters:

$$
\begin{aligned}
\delta b_{i}^{\mu} & =\xi_{i} v_{i}^{\mu}+i \bar{\epsilon}_{i} \Psi_{i}^{\mu}+i \epsilon_{i} \bar{\Psi}_{i}^{\mu}, \\
\delta v_{i}^{\mu} & =0 \\
\delta \Psi_{i}^{\mu} & =-\epsilon_{i} v_{i}^{\mu}-i \alpha_{i} \Psi_{i}^{\mu}, \\
\delta \bar{\Psi}_{i}^{\mu} & =-\bar{\epsilon}_{i} v_{i}^{\mu}+i \alpha_{i} \bar{\Psi}_{i}^{\mu},
\end{aligned}
$$

\footnotetext{
${ }^{4}$ Fermionic derivatives act to the right.
} 
for constant shift parameters $\xi_{i}, \epsilon_{i}, \bar{\epsilon}_{i}$ and $\alpha_{i}$. Hence the eikonal phase $\chi$ depending only on the background parameters will be invariant under

$$
\delta \chi=\frac{\partial \chi}{\partial b_{i}^{\mu}} \delta b_{i}^{\mu}+\frac{\partial \chi}{\partial \Psi_{i}^{\mu}} \delta \Psi_{i}^{\mu}+\frac{\partial \chi}{\partial \bar{\Psi}_{i}^{\mu}} \delta \bar{\Psi}_{i}^{\mu}=0
$$

for both $i=1,2$. For each parameter we recover a constraint:

$$
\begin{aligned}
\xi_{i}: & 0=v_{i}^{\mu}\left\langle\Delta p_{i, \mu}\right\rangle, \\
\epsilon_{i}: & 0=\left\langle\Delta p_{i, \mu}\right\rangle \bar{\Psi}_{i}^{\mu}+m_{i} v_{i}^{\mu}\left\langle\Delta \bar{\psi}_{i, \mu}\right\rangle, \\
\bar{\epsilon}_{i}: & 0=\left\langle\Delta p_{i, \mu}\right\rangle \Psi_{i}^{\mu}+m_{i} v_{i}^{\mu}\left\langle\Delta \psi_{i, \mu}\right\rangle, \\
\alpha_{i}: & 0=\bar{\Psi}_{i}^{\mu}\left\langle\Delta \psi_{i, \mu}\right\rangle+\left\langle\Delta \bar{\psi}_{i, \mu}\right\rangle \Psi_{i}^{\mu} .
\end{aligned}
$$

These four constraints respectively imply conservation of $p_{i}^{2}, p_{i} \cdot \bar{\psi}_{i}, p_{i} \cdot \psi_{i}$ and $\psi_{i} \cdot \bar{\psi}_{i}$ between initial and final asymptotic states, i.e. the energy/mass, conserved supercharges and spin length. ${ }^{5}$

How do we re-interpret the latter three constraints in terms of the classical spin tensors $S_{i}^{\mu \nu}$ ? Using $\left\langle\Delta S_{i}^{\mu \nu}\right\rangle=-2 i \bar{\Psi}_{i}^{[\mu}\left\langle\Delta \psi_{i}^{\nu]}\right\rangle-2 i\left\langle\Delta \bar{\psi}_{i}^{[\mu}\right\rangle \Psi_{i}^{\nu]}$ we have

$$
\begin{aligned}
m_{i} v_{i, \mu}\left\langle\Delta S_{i}^{\mu \nu}\right\rangle+\left\langle\Delta p_{i, \mu}\right\rangle S_{i}^{\mu \nu}= & -i\left(\left\langle\Delta p_{i, \mu}\right\rangle \bar{\Psi}_{i}^{\mu}+m_{i} v_{i}^{\mu}\left\langle\Delta \bar{\psi}_{i, \mu}\right\rangle\right) \Psi_{i}^{\nu} \\
& -i\left(\left\langle\Delta p_{i, \mu}\right\rangle \Psi_{i}^{\mu}+m_{i} v_{i}^{\mu}\left\langle\Delta \psi_{i, \mu}\right\rangle\right) \bar{\Psi}_{i}^{\nu} \\
& -i m_{i}\left(v_{i} \cdot \bar{\Psi}_{i}\left\langle\Delta \psi_{i}^{\nu}\right\rangle+v_{i} \cdot \Psi_{i}\left\langle\Delta \bar{\psi}_{i}^{\nu}\right\rangle\right) .
\end{aligned}
$$

Therefore, $p_{i, \mu} S_{i}^{\mu \nu}$ is conserved only if, in addition to eqs. (4.34b) and (4.34c), we choose $v_{i} \cdot \Psi_{i}=v_{i} \cdot \bar{\Psi}_{i}=0$. This is consistent with our observation in section 3 that the $\mathcal{N}=2$ SUSY theory agrees with the spinning particle action only when we use the covariant SSC: $\pi_{i, \mu} S_{i}^{\mu \nu}=0$, which is implied by $\pi_{i} \cdot \psi_{i}=\pi_{i} \cdot \bar{\psi}_{i}=0$. Meanwhile,

$$
\mathcal{S}_{i, \mu \nu}\left\langle\Delta S_{i}^{\mu \nu}\right\rangle=2 \Psi_{i} \cdot \bar{\Psi}_{i}\left(\bar{\Psi}_{i}^{\mu}\left\langle\Delta \psi_{i, \mu}\right\rangle+\left\langle\Delta \bar{\psi}_{i, \mu}\right\rangle \Psi_{i}^{\mu}\right),
$$

so preservation of the spin lengths $\operatorname{tr}\left(S_{i} \cdot S_{i}\right)=-2 s_{i}^{2}$ is guaranteed by eq. (4.34d).

One should note that the background symmetries (4.32) are gauge fixed by our previous requirements that $b \cdot v_{i}=0$ and $v_{i} \cdot \Psi_{i}=v_{i} \cdot \bar{\Psi}_{i}=0$, the latter implying $v_{i, \mu} \mathcal{S}_{i}^{\mu \nu}=0$. In terms of the shifts (4.32) these constraints are achieved using

$$
\begin{aligned}
& \epsilon_{i}=v_{i} \cdot \Psi_{i}, \quad \bar{\epsilon}_{i}=v_{i} \cdot \bar{\Psi}_{i}, \quad \alpha_{i}=0, \\
& \xi_{1}=\frac{b \cdot\left(\gamma v_{2}-v_{1}\right)-v_{1} \cdot \mathcal{S}_{2} \cdot v_{2}+\gamma v_{1} \cdot \mathcal{S}_{1} \cdot v_{2}}{\gamma^{2}-1}, \\
& \xi_{2}=\frac{b \cdot\left(v_{2}-\gamma v_{1}\right)+v_{1} \cdot \mathcal{S}_{1} \cdot v_{2}-\gamma v_{1} \cdot \mathcal{S}_{2} \cdot v_{1}}{\gamma^{2}-1} .
\end{aligned}
$$

However, no information is lost: full dependence on terms of the form $b \cdot v_{i}$ and $v_{i, \mu} \mathcal{S}_{i}^{\mu \nu}$ is restored to the eikonal phase by shifting

$$
\begin{aligned}
\mathcal{S}_{i}^{\mu \nu} & \rightarrow \mathcal{S}_{i}^{\mu \nu}+2\left(v_{i} \cdot \mathcal{S}_{i}\right)^{[\mu} v_{i}^{\nu]} \\
b^{\mu} & \rightarrow b^{\mu}+\xi_{2} v_{2}^{\mu}-\xi_{1} v_{1}^{\mu}+\mathcal{S}_{2}^{\mu \nu} v_{2, \nu}-\mathcal{S}_{1}^{\mu \nu} v_{1, \nu},
\end{aligned}
$$

with $\xi_{i}$ as given above.

\footnotetext{
${ }^{5}$ Although the true supercharges $Q_{i}=\pi_{i} \cdot \psi_{i}, \bar{Q}_{i}=\pi_{i} \cdot \bar{\psi}_{i}$ involve the covariantized momentum $\pi_{i, \mu}=$ $p_{i, \mu}-i \omega_{\mu a b} \bar{\psi}_{i}^{a} \psi_{i}^{b}$, the spacetime is asymptotically flat so $\omega_{\mu a b}=0$ at the boundary.
} 
Given our imposition of these background constraints, in order to truly "check" the background symmetries of the eikonal phase one should calculate it without making these requirements a priori. Up to the $2 \mathrm{PM}$ order described in section 5 we have done so, as a separate calculation which involved generalizing the Feynman rules in section 4.1 to the inclusion of such terms. Notice also that the background field symmetries (4.32) continue to apply when $C_{E, i} \neq 0$ : although local SUSY is spoiled by the presence of additional curvature couplings in the action, approximate SUSY persists up to spin-squared effects as discussed in section 3.3. So we continue to expect conservation of energy, spin length, and the SSC. In ref. [2] the same approximate SUSY was also seen acting on the leading-PM waveform $\left\langle h_{\mu \nu}(k)\right\rangle$.

\section{The eikonal phase and derived observables}

In this section we compute the eikonal phase $\chi=-i \log \mathcal{Z}_{\text {WQFT }}$ up to $2 \mathrm{PM}$ order, and from it derive the momentum impulse $\left\langle\Delta p_{i}^{\mu}\right\rangle$, spin kick $\left\langle\Delta S_{i}^{\mu \nu}\right\rangle$ and aligned-spin scattering angle $\theta$ using the relationships established in section 4.3.

\section{$5.12 \mathrm{PM}$ eikonal phase}

Up to $2 \mathrm{PM}$ order the eikonal phase is given as the sum of four vacuum diagrams in the WQFT:

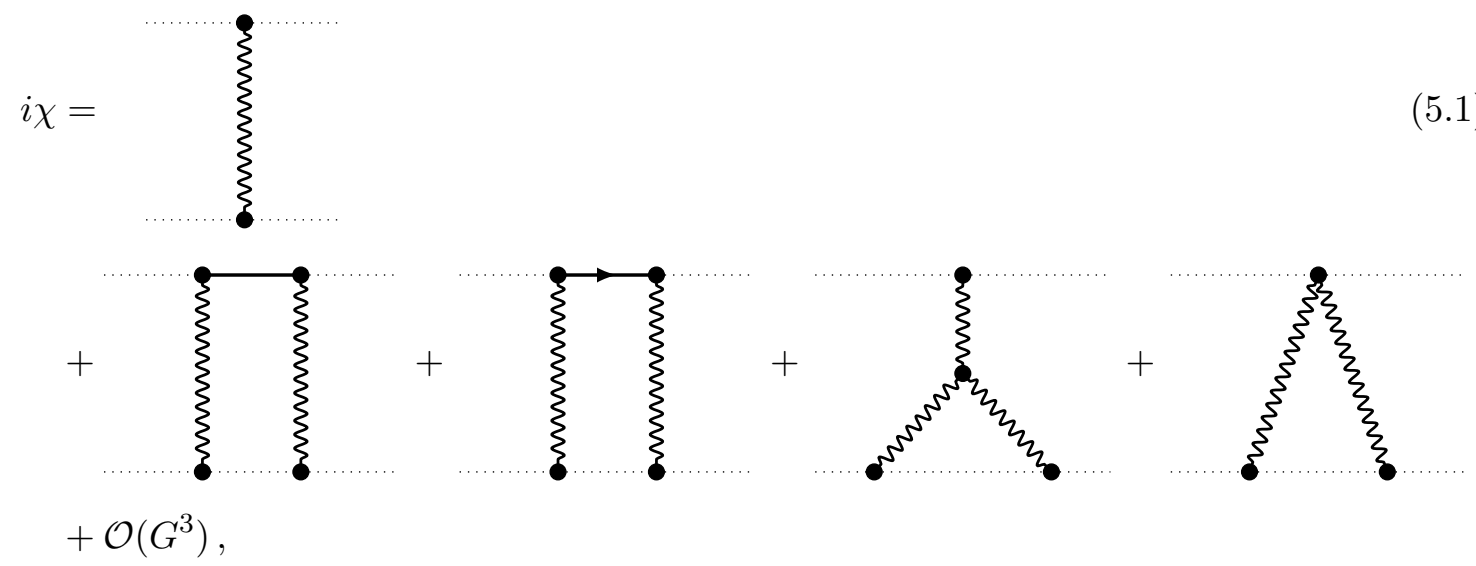

where mirror diagrams $(1 \leftrightarrow 2)$ are left implicit and we sum over both directions of the arrowed line (representing a propagating spin mode $\psi_{i}^{\prime a}$ ). Explicit expressions are obtained using the Feynman rules given in section 4.1. For example, the 1PM contribution only involves the graviton source vertex (4.18) and has the explicit form

$$
\begin{aligned}
& \sum_{2 \uparrow}=i \frac{\kappa^{2} m_{1} m_{2}}{4} \int_{q} e^{i q \cdot b} \delta\left(q \cdot v_{1}\right) \delta\left(q \cdot v_{2}\right) \frac{P_{\mu \nu ; \rho \sigma}}{q^{2}+i \epsilon} \\
& \times\left(v_{1}^{\mu} v_{1}^{\nu}-i\left(q \cdot \mathcal{S}_{1}\right)^{\mu} v_{1}^{\nu}-\frac{1}{2}\left(q \cdot \mathcal{S}_{1}\right)^{\mu}\left(q \cdot \mathcal{S}_{1}\right)^{\nu}+\frac{C_{E, 1}}{2} v_{1}^{\mu} v_{1}^{\nu}\left(q \cdot \mathcal{S}_{1} \cdot \mathcal{S}_{1} \cdot q\right)\right) \\
& \times\left(v_{2}^{\rho} v_{2}^{\sigma}+i\left(q \cdot \mathcal{S}_{2}\right)^{\rho} v_{2}^{\sigma}-\frac{1}{2}\left(q \cdot \mathcal{S}_{2}\right)^{\rho}\left(q \cdot \mathcal{S}_{2}\right)^{\sigma}+\frac{C_{E, 2}}{2} v_{2}^{\rho} v_{2}^{\sigma}\left(q \cdot \mathcal{S}_{2} \cdot \mathcal{S}_{2} \cdot q\right)\right),
\end{aligned}
$$


where we discard all terms above $\mathcal{O}\left(\mathcal{S}^{2}\right) ; b^{\mu}=b_{2}^{\mu}-b_{1}^{\mu}$ and we integrate over the offshell momentum $q$ of the exchanged graviton. Similar expressions are easily assembled for the other diagrams; for a worldline propagator of either type $\left(z^{\mu}\right.$ or $\left.\psi^{\prime \mu}\right)$ we perform a one-dimensional integral $\int_{\omega}$ over the intermediate energy $\omega$.

An important practical consideration is our use of the constant spinors $\Psi_{i}^{\mu}$ : in general, we prefer final results to be expressed in terms of the physically relevant antisymmetric spin tensors $\mathcal{S}_{i}^{\mu \nu}=-2 i \bar{\Psi}_{i}^{[\mu} \Psi_{i}^{\nu]}$. This motivates our writing the interaction vertices (4.18)-(4.21) in terms of $\mathcal{S}^{\mu \nu}$ wherever possible - so that most of the graphs in eq. (5.1) depend on $\Psi_{i}^{\mu}$ only via $\mathcal{S}_{i}^{\mu \nu}$. The only exception is the third diagram, which carries an overall factor $\bar{\Psi}_{1}^{\mu} \Psi_{1}^{\nu}$ due to the spinor vertex (4.21) (appropriately contracted with the rest of the expression). Manifest dependence on $\mathcal{S}_{1}^{\mu \nu}$ is only recovered once the counterpart diagram with the arrowed line pointing in the opposite direction is included: except for its overall dependence on $\Psi_{1}^{\mu}$ the expression is identical, and we recover $\bar{\Psi}_{1}^{\mu} \Psi_{1}^{\nu}+\Psi_{1}^{\mu} \bar{\Psi}_{1}^{\nu}=i \mathcal{S}_{1}^{\mu \nu}$ as an overall factor.

As the techniques used to integrate these expressions are now well-established (see e.g. refs. $[38,110]$ ) we relegate those details to appendix $\mathrm{B}$, and here simply present our results. As explained in section $4.3, \chi=\sum_{n=1}^{\infty} G^{n} \chi^{(n)}$ depends only on the orthogonal components of $b^{\mu}$ and $\mathcal{S}_{i}^{\mu \nu}$ with respect to the velocities $v_{i}^{\mu}$, so we set $b \cdot v_{i}=0$ and $\left(v_{i} \cdot \mathcal{S}_{i}\right)^{\mu}=0$ (the covariant SSC) without loss of generality. At 1PM order the various $D$-dimensional contributions are ${ }^{6}$

$$
\begin{aligned}
\left.\chi^{(1)}\right|_{\mathcal{S}_{1}^{0} \mathcal{S}_{2}^{0}}= & \frac{2 \pi^{2-\frac{D}{2}} \Gamma\left(\frac{D}{2}-2\right)\left((D-2) \gamma^{2}-1\right) m_{1} m_{2}}{(D-2)|b|^{D-4} \sqrt{\gamma^{2}-1}} \\
\left.\chi^{(1)}\right|_{\mathcal{S}_{1} \mathcal{S}_{2}^{0}}= & \frac{4 \pi^{2-\frac{D}{2}} \Gamma\left(\frac{D}{2}-1\right) \gamma m_{1} m_{2}}{|b|^{D-3} \sqrt{\gamma^{2}-1} \cdot \mathcal{S}_{1} \cdot v_{2}} \\
\left.\chi^{(1)}\right|_{\mathcal{S}_{1} \mathcal{S}_{2}=}= & \frac{2 \pi^{2-\frac{D}{2}} \Gamma\left(\frac{D}{2}-1\right) m_{1} m_{2}}{|b|^{D-2}\left(\gamma^{2}-1\right)^{3 / 2}} \\
& \times\left(\left(\gamma^{2}-1\right)\left(\gamma \operatorname{tr}\left(\mathcal{S}_{1} \cdot \mathcal{S}_{2}\right)-(D-2)\left(\hat{b} \cdot \mathcal{S}_{1} \cdot v_{2} \hat{b} \cdot \mathcal{S}_{2} \cdot v_{1}-\gamma \hat{b} \cdot \mathcal{S}_{1} \cdot \mathcal{S}_{2} \cdot \hat{b}\right)\right)\right. \\
\left.\chi^{(1)}\right|_{\mathcal{S}_{1}^{2} \mathcal{S}_{2}^{0}=} & \frac{2 \pi^{2-\frac{D}{2}} \Gamma\left(\frac{D}{2}-1\right) m_{1} m_{2}}{(D-2)|b|^{D-2}\left(\gamma^{2}-1\right)^{3 / 2}} \\
& \times\left(\left(\gamma^{2}-1\right)\left((D-2)^{2}\left(\hat{b} \cdot \mathcal{S}_{1} \cdot v_{2}\right)^{2}+(D-2) \hat{b} \cdot \mathcal{S}_{1} \cdot \mathcal{S}_{1} \cdot \hat{b}-2 s_{1}^{2}\right)\right. \\
& +\left(D-1-\mathcal{S}^{2}(D-2)\right) v_{2} \cdot \mathcal{S}_{1} \cdot \mathcal{S}_{1} \cdot v_{2} \\
& \quad-C_{E, 1}\left((D-2) \gamma^{2}-1\right)\left(\left(\gamma^{2}-1\right)\left((D-2) \hat{b} \cdot \mathcal{S}_{1} \cdot \mathcal{S}_{1} \cdot \hat{b}-2 s_{1}^{2}\right)\right. \\
& \left.\left.+v_{2} \cdot \mathcal{S}_{1} \cdot \mathcal{S}_{1} \cdot v_{2}\right)\right)
\end{aligned}
$$

where $\hat{b}^{\mu}:=b^{\mu} /|b|,|b|=\sqrt{-b \cdot b}$ and we recall that $\gamma=v_{1} \cdot v_{2}$. As the $2 \mathrm{PM}$ results are

\footnotetext{
${ }^{6}$ The zeroth-order-spin contribution (5.3a) is logarithmically divergent in $D=4$ dimensions - this pole is unphysical and affects neither the impulse nor spin kick.
} 
more involved we provide them here only in $D=4$ dimensions:

$$
\begin{aligned}
\left.\chi^{(2)}\right|_{\mathcal{S}_{1}^{0} \mathcal{S}_{2}^{0}}= & \frac{3 \pi\left(5 \gamma^{2}-1\right)\left(m_{1}+m_{2}\right) m_{1} m_{2}}{4|b| \sqrt{\gamma^{2}-1}} \\
\left.\chi^{(2)}\right|_{\mathcal{S}_{1} \mathcal{S}_{2}^{0}}= & \frac{\pi \gamma\left(5 \gamma^{2}-3\right)\left(4 m_{1}+3 m_{2}\right) m_{1} m_{2}}{4|b|^{2}\left(\gamma^{2}-1\right)^{3 / 2}} \hat{b} \cdot \mathcal{S}_{1} \cdot v_{2} \\
\left.\chi^{(2)}\right|_{\mathcal{S}_{1} \mathcal{S}_{2}}= & \frac{\pi\left(m_{1}+m_{2}\right) m_{1} m_{2}}{4|b|^{3}\left(\gamma^{2}-1\right)^{5 / 2}} \\
& \times\left(( \gamma ^ { 2 } - 1 ) \left(\gamma\left(5 \gamma^{2}-3\right)\left(2 \operatorname{tr}\left(\mathcal{S}_{1} \cdot \mathcal{S}_{2}\right)+3 \hat{b} \cdot \mathcal{S}_{1} \cdot \mathcal{S}_{2} \cdot \hat{b}\right)\right.\right. \\
& \left.\left.\quad-9\left(5 \gamma^{2}-1\right) \hat{b} \cdot \mathcal{S}_{1} \cdot v_{2} \hat{b} \cdot \mathcal{S}_{2} \cdot v_{1}\right)-3\left(3 \gamma^{2}-1\right) v_{2} \cdot \mathcal{S}_{1} \cdot \mathcal{S}_{2} \cdot v_{1}\right) \\
\left.\chi^{(2)}\right|_{\mathcal{S}_{1}^{2} \mathcal{S}_{2}^{0}} & =\frac{\pi m_{1} m_{2}}{64|b|^{3}\left(\gamma^{2}-1\right)^{5 / 2}} \\
& \quad\left(8\left(\gamma^{2}-1\right)\left(\left(13 \gamma^{4}-42 \gamma^{2}+21\right) m_{1}-4\left(3 \gamma^{2}-1\right) m_{2}\right) s_{1}^{2}\right. \\
& \quad-6\left(\gamma^{2}-1\right)\left(\left(29 \gamma^{4}-66 \gamma^{2}+29\right) m_{1}-4\left(3 \gamma^{2}-1\right) m_{2}\right) \hat{b} \cdot \mathcal{S}_{1} \cdot \mathcal{S}_{1} \cdot \hat{b} \\
& +24\left(\gamma^{2}-1\right)\left(\left(31 \gamma^{2}-11\right) m_{1}+3\left(5 \gamma^{2}-1\right) m_{2}\right)\left(\hat{b} \cdot \mathcal{S}_{1} \cdot v_{2}\right)^{2} \\
& -6\left(\left(49 \gamma^{4}-90 \gamma^{2}+33\right) m_{1}+4\left(5 \gamma^{4}-9 \gamma^{2}+2\right) m_{2}\right) v_{2} \cdot \mathcal{S}_{1} \cdot \mathcal{S}_{1} \cdot v_{2} \\
& +4 C_{E, 1}\left(\gamma^{2}-1\right)\left(\left(125 \gamma^{4}-138 \gamma^{2}+29\right) m_{1}+2\left(45 \gamma^{4}-42 \gamma^{2}+5\right) m_{2}\right) s_{1}^{2} \\
& -3 C_{E, 1}\left(\gamma^{2}-1\right)\left(\left(155 \gamma^{4}-174 \gamma^{2}+35\right) m_{1}+4\left(30 \gamma^{4}-29 \gamma^{2}+3\right) m_{2}\right) \hat{b} \cdot \mathcal{S}_{1} \cdot \mathcal{S}_{1} \cdot \hat{b} \\
& \left.\left.-3 C_{E, 1}\left(\left(95 \gamma^{4}-102 \gamma^{2}+23\right) m_{1}+4\left(15 \gamma^{4}-13 \gamma^{2}+2\right) m_{2}\right)\right) v_{2} \cdot \mathcal{S}_{1} \cdot \mathcal{S}_{1} \cdot v_{2}\right)
\end{aligned}
$$

We have confirmed agreement between these four-dimensional results and refs. [55, 62]. ${ }^{7}$ For the full $D$-dimensional expressions at $2 \mathrm{PM}$ order we refer the reader to the supplementary material of this paper.

\section{$5.2 \quad 1 P M$ observables}

The impulse, spin kick and aligned-spin scattering angle are derived from the eikonal phase $\chi$ using

$$
\left\langle\Delta p_{i, \mu}\right\rangle=-\frac{\partial \chi}{\partial b_{i}^{\mu}}, \quad\left\langle\Delta S_{i}^{\mu \nu}\right\rangle=\frac{4}{m_{i}} \mathcal{S}_{i}^{\rho[\mu} \frac{\partial \chi}{\partial \mathcal{S}_{i, \nu]}^{\rho}}, \quad \sin \left(\frac{\theta}{2}\right)=-\frac{1}{2 p_{\infty}} \frac{\partial \chi}{\partial|b|},
$$

where $p_{\infty}$ is the centre-of-mass momentum (4.30). Care should be taken with these derivatives as the 1PM eikonal phase given in eq. (5.3) depends only on the $(D-2)$-dimensional orthogonal components of $b^{\mu}=b_{2}^{\mu}-b_{1}^{\mu}$ and $\mathcal{S}_{i}^{\mu \nu}$ with respect to the velocities $v_{i}^{\mu}$. As explained in section 4.3, full dependence on terms of the form $b \cdot v_{i}$ and $\left(v_{i} \cdot \mathcal{S}_{i}\right)^{\mu}$ is restored to the eikonal phase using the SUSY shifts in eq. (4.38), after which the derivatives (5.5) may be taken without issue. One may then safely re-impose $b \cdot v_{i}=0$ and $\left(v_{i} \cdot \mathcal{S}_{i}\right)^{\mu}=0$ on the resulting physical observables. Notice that eq. (5.5) implies conservation of momentum $\left\langle\Delta p_{2}^{\mu}\right\rangle=-\left\langle\Delta p_{1}^{\mu}\right\rangle$ as all dependence on $b_{i}^{\mu}$ comes via the relative impact parameter $b^{\mu}=b_{2}^{\mu}-b_{1}^{\mu}$ (we are free to choose a spacetime origin). At higher-PM orders this implies

\footnotetext{
${ }^{7}$ We find it simplest to compare our expressions with covariant scattering amplitudes before the Fourier transform with respect to $q^{\mu}$ is taken.
} 
that the scattering is conservative, i.e. by this procedure we miss radiation-reaction effects for which $\left\langle\Delta p_{2}^{\mu}\right\rangle \neq-\left\langle\Delta p_{1}^{\mu}\right\rangle$.

The $\mathcal{O}(G)$ part of the impulse $\left\langle\Delta p_{1}^{\mu}\right\rangle=\sum_{n=1}^{\infty} G^{n}\left\langle\Delta p_{1}^{\mu}\right\rangle^{(n)}$ is given by

$$
\begin{aligned}
& \left.\left\langle\Delta p_{1}^{\mu}\right\rangle^{(1)}\right|_{\mathcal{S}_{1}^{0} \mathcal{S}_{2}^{0}}=\frac{4 \pi^{2-\frac{D}{2}} \Gamma\left(\frac{D}{2}-1\right)\left((D-2) \gamma^{2}-1\right) m_{1} m_{2}}{(D-2)|b|^{D-3} \sqrt{\gamma^{2}-1}} \hat{b}^{\mu} \\
& \left.\left\langle\Delta p_{1}^{\mu}\right\rangle^{(1)}\right|_{\mathcal{S}_{1} \mathcal{S}_{2}^{0}}=-\frac{4 \pi^{2-\frac{D}{2}} \Gamma\left(\frac{D}{2}-1\right) \gamma m_{1} m_{2}}{|b|^{D-2} \sqrt{\gamma^{2}-1}}\left((D-2) \hat{b} \cdot \mathcal{S}_{1} \cdot v_{2} \hat{b}^{\mu}+\left(\mathcal{S}_{1} \cdot v_{2}\right)^{\mu}\right) \\
& \left.\left\langle\Delta p_{1}^{\mu}\right\rangle^{(1)}\right|_{\mathcal{S}_{1} \mathcal{S}_{2}}=\frac{4 \pi^{2-\frac{D}{2}} \Gamma\left(\frac{D}{2}\right) m_{1} m_{2}}{|b|^{D-1} \sqrt{\gamma^{2}-1}}\left(\hat{b} \cdot \mathcal{S}_{1} \cdot v_{2}\left(v_{1} \cdot \mathcal{S}_{2}\right)^{\mu}+\hat{b} \cdot \mathcal{S}_{2} \cdot v_{1}\left(v_{2} \cdot \mathcal{S}_{1}\right)^{\mu}\right. \\
& \quad+\left(\gamma D \hat{b} \cdot \mathcal{S}_{1} \cdot \mathcal{S}_{2} \cdot \hat{b}-D \hat{b} \cdot \mathcal{S}_{1} \cdot v_{2} \hat{b} \cdot \mathcal{S}_{2} \cdot v_{1}+\gamma \operatorname{tr}\left(\mathcal{S}_{1} \cdot \mathcal{S}_{2}\right)-\frac{v_{2} \cdot \mathcal{S}_{1} \cdot \mathcal{S}_{2} \cdot v_{1}}{\gamma^{2}-1}\right) \hat{b}^{\mu} \\
& \left.\quad+\gamma\left(\hat{b} \cdot \mathcal{S}_{1} \cdot \mathcal{S}_{2} \cdot P_{12}\right)^{\mu}+\gamma\left(P_{12} \cdot \mathcal{S}_{1} \cdot \mathcal{S}_{2} \cdot \hat{b}\right)^{\mu}\right) \\
& \quad+\left((D-2) D\left(\hat{b} \cdot \mathcal{S}_{1} \cdot v_{2}\right)^{2}+D \hat{b} \cdot \mathcal{S}_{1} \cdot \mathcal{S}_{1} \cdot \hat{b}+\frac{D-1-(D-2) \gamma^{2}}{\gamma_{1}-1} v_{2} \cdot \mathcal{S}_{1} \cdot \mathcal{S}_{1} \cdot v_{2}\right. \\
& \quad-2 v_{\mathcal{S}_{1}^{2} \mathcal{S}_{2}^{0}}=\frac{2 \pi^{2-\frac{D}{2}} \Gamma\left(\frac{D}{2}-1\right) m_{1} m_{2}}{|b|^{D-1} \sqrt{\gamma^{2}-1}}\left(-2(D) \mathcal{S}_{1}\right)^{\mu} \\
& \left.\left.\quad+\left(D \hat{b} \cdot \hat{S}_{1} \cdot \mathcal{S}_{1} \cdot \hat{b}+\operatorname{tr}\left(\mathcal{S}_{1} \cdot P_{12} \cdot \mathcal{S}_{1}\right)\right) \hat{b}^{\mu}\right)\right)
\end{aligned}
$$

and $\left\langle\Delta p_{2}^{\mu}\right\rangle=-\left\langle\Delta p_{1}^{\mu}\right\rangle$. These expressions are manifestly orthogonal to $v_{i}^{\mu}$, as required by eq. (4.34a), which is apparent given our use of the projector $P_{12}^{\mu \nu}$ to the $(D-2)$-dimensional space orthogonal to these velocities:

$$
P_{12}^{\mu \nu}=\eta^{\mu \nu}+\frac{1}{\gamma^{2}-1}\left[v_{1}^{\mu} v_{1}^{\nu}-2 \gamma v_{1}^{(\mu} v_{2}^{\nu)}+v_{2}^{\mu} v_{2}^{\nu}\right] .
$$

The $\mathcal{O}(G)$ part of the spin kick $\left\langle\Delta S_{1}^{\mu \nu}\right\rangle=\sum_{n=1}^{\infty} G^{n}\left\langle\Delta S_{1}^{\mu \nu}\right\rangle^{(n)}$ is

$$
\begin{aligned}
& \left.\left\langle\Delta S_{1}^{\mu \nu}\right\rangle^{(1)}\right|_{\mathcal{S}_{1}^{1} \mathcal{S}_{2}^{0}}=-\frac{8 \pi^{2-\frac{D}{2}} \Gamma\left(\frac{D}{2}-1\right) m_{2}}{|b|^{D-3} \sqrt{\gamma^{2}-1}} \\
& \quad \times\left(\frac{\left(\hat{b} \cdot \mathcal{S}_{1}\right)^{[\mu} v_{1}^{\nu]}}{D-2}+\gamma\left(v_{2} \cdot \mathcal{S}_{1}\right)^{[\mu} \hat{b}^{\nu]}-\gamma\left(\hat{b} \cdot \mathcal{S}_{1}\right)^{[\mu} v_{2}^{\nu]}\right) \\
& \left.\left\langle\Delta S_{1}^{\mu \nu}\right\rangle^{(1)}\right|_{\mathcal{S}_{1}^{2} \mathcal{S}_{2}^{0}}=\frac{8 \pi^{2-\frac{D}{2}} \Gamma\left(\frac{D}{2}-1\right) m_{2}}{|b|^{D-2} \sqrt{\gamma^{2}-1}} \\
& \quad \times\left(-\left(\mathcal{S}_{1} \cdot \mathcal{S}_{1} \cdot \hat{b}\right)^{[\mu} \hat{b}^{\nu]}-(D-2) \hat{b} \cdot \mathcal{S}_{1} \cdot v_{2}\left(\left(\mathcal{S}_{1} \cdot v_{2}\right)^{\left[\mu \hat{b}^{\nu]}\right.}-\left(\mathcal{S}_{1} \cdot \hat{b}\right)^{[\mu} v_{2}{ }^{\nu]}\right)\right. \\
& \quad+\frac{\gamma\left(\mathcal{S}_{1} \cdot \mathcal{S}_{1} \cdot v_{2}\right)^{[\mu} v_{1}{ }^{\nu]}}{\left(\gamma^{2}-1\right)(D-2)}+\frac{1+(D-2) \gamma^{2}-D}{\left(\gamma^{2}-1\right)(D-2)}\left(\mathcal{S}_{1} \cdot \mathcal{S}_{1} \cdot v_{2}\right)^{[\mu} v_{2}{ }^{\nu]} \\
& \left.\quad+C_{E, 1}\left((D-2) \gamma^{2}-1\right)\left(\left(\mathcal{S}_{1} \cdot \mathcal{S}_{1} \cdot \hat{b}\right)^{[\mu} \hat{b}^{\nu]}-\frac{\left(\mathcal{S}_{1} \cdot \mathcal{S}_{1} \cdot v_{2}\right)^{[\mu}\left(\gamma v_{1}-v_{2}\right)^{\nu]}}{\left(\gamma^{2}-1\right)(D-2)}\right)\right)
\end{aligned}
$$




$$
\begin{aligned}
& \left.\left\langle\Delta S_{1}^{\mu \nu}\right\rangle^{(1)}\right|_{\mathcal{S}_{1}^{1} \mathcal{S}_{2}^{1}}=\frac{4 \pi^{2-\frac{D}{2}} \Gamma\left(\frac{D}{2}-1\right) m_{2}}{|b|^{D-2} \sqrt{\gamma^{2}-1}} \\
& \times\left(\gamma(D-2)\left(\left(\mathcal{S}_{1} \cdot \hat{b}\right)^{[\mu}\left(\mathcal{S}_{2} \cdot \hat{b}\right)^{\nu]}-\left(\mathcal{S}_{1} \cdot \mathcal{S}_{2} \cdot \hat{b}\right)^{[\mu} \hat{b}^{\nu]}\right)-\frac{\left(\mathcal{S}_{1} \cdot v_{2}\right)^{[\mu}\left(\mathcal{S}_{2} \cdot v_{1}\right)^{\nu]}}{\gamma^{2}-1}\right. \\
& \quad-\frac{\left(\mathcal{S}_{1} \cdot \mathcal{S}_{2} \cdot v_{1}\right)^{[\mu}\left(\gamma v_{1}-v_{2}\right)^{\nu]}}{\gamma^{2}-1}-2 \gamma\left(\mathcal{S}_{1} \cdot \mathcal{S}_{2}\right)^{[\mu \nu]} \\
& \left.\left.\quad-(D-2)\left(\left(\mathcal{S}_{1} \cdot \hat{b}\right)^{[\mu} v_{2}^{\nu]}-\left(\mathcal{S}_{1} \cdot v_{2}\right)^{[\mu} \hat{b} \nu\right]\right) \hat{b} \cdot \mathcal{S}_{2} \cdot v_{1}\right)
\end{aligned}
$$

The other components are $\left.\left\langle\Delta \mathcal{S}_{1}^{\mu \nu}\right\rangle^{(1)}\right|_{\mathcal{S}_{1}^{0} \mathcal{S}_{2}^{1}}=\left.\left\langle\Delta \mathcal{S}_{1}^{\mu \nu}\right\rangle^{(1)}\right|_{\mathcal{S}_{1}^{0} \mathcal{S}_{2}^{2}}=0 ;\left\langle\Delta \mathcal{S}_{2}^{\mu \nu}\right\rangle^{(1)}$ is recovered by simple relabelling. Finally, the $\mathcal{O}(G)$ part of the scattering angle $\theta=\sum_{n=1}^{\infty} G^{n} \theta^{(n)}$ emerging in the case of aligned spins is

$$
\begin{aligned}
\theta^{(1)}=\frac{2 \pi^{2-\frac{D}{2}}(D-3) \Gamma\left(\frac{D}{2}-1\right) E}{|b|^{D-3}\left(\gamma^{2}-1\right)}\left(\frac{2(D-2) \gamma^{2}-2}{(D-3)(D-2)}+2 \gamma \sqrt{\gamma^{2}-1} \frac{s_{1}+s_{2}}{|b|}\right. \\
+\left((D-2) \gamma^{2}-\frac{3}{4} D+2\right) \frac{\left(s_{1}+s_{2}\right)^{2}}{|b|^{2}}-\frac{D-4}{4} \frac{\left(s_{1}-s_{2}\right)^{2}}{|b|^{2}} \\
\left.\quad-\left((D-2) \gamma^{2}-1\right)\left(\frac{C_{E, 1} s_{1}^{2}+C_{E, 2} s_{2}^{2}}{|b|^{2}}\right)\right) .
\end{aligned}
$$

To specify aligned spins we have inserted

$$
\mathcal{S}_{1}^{\mu \nu}=2 s_{1} \frac{b^{[\mu}\left(\gamma v_{1}-v_{2}\right)^{\nu]}}{|b| \sqrt{\gamma^{2}-1}}, \quad \mathcal{S}_{2}^{\mu \nu}=2 s_{2} \frac{b^{[\mu}\left(v_{1}-\gamma v_{2}\right)^{\nu]}}{|b| \sqrt{\gamma^{2}-1}}
$$

with the normalizations ensuring that $\operatorname{tr}\left(\mathcal{S}_{i} \cdot \mathcal{S}_{i}\right)=-2 s_{i}^{2}$. The aligned-spin tensors live in the subspace spanned by $b^{\mu}, v_{1}^{\mu}$ and $v_{2}^{\mu}$, which together with the $\operatorname{SSC}\left(v_{i} \cdot \mathcal{S}_{i}\right)^{\mu}=0$ defines them uniquely. This definition ensures planar dynamics and includes the conventional definition in four spacetime dimensions.

\section{$5.3 \quad 2 \mathrm{PM}$ observables}

The 2PM momentum impulse and spin kick are again derived from the eikonal phase by taking derivatives with respect to the background parameters $b_{i}^{\mu}$ and $\mathcal{S}_{i}^{\mu \nu}(5.5)$; however, an additional subtlety is the interpretation of these background parameters (and $v_{i}^{\mu}$ ). In general, we prefer to express observables in terms of $b_{ \pm \infty}^{\mu}, v_{i, \pm \infty}^{\mu}$ and $\mathcal{S}_{i, \pm \infty}^{\mu}$ taken in the far past or future:

$$
\begin{aligned}
& x_{i}^{\mu}(\tau) \underset{\tau \rightarrow \pm \infty}{\longrightarrow} b_{i, \pm \infty}^{\mu}+\tau v_{i, \pm \infty}^{\mu}, \\
& S_{i}^{\mu \nu}(\tau) \underset{\tau \rightarrow \pm \infty}{\longrightarrow} \mathcal{S}_{i, \pm \infty}^{\mu \nu} .
\end{aligned}
$$

With the time-symmetric worldline propagators (4.15) the currently used background parameters are $b^{\mu}=\frac{1}{2}\left(b_{+\infty}^{\mu}+b_{-\infty}^{\mu}\right)+\mathcal{O}\left(G^{2}\right), v_{i}^{\mu}=\frac{1}{2}\left(v_{i,+\infty}^{\mu}+v_{i,-\infty}^{\mu}\right)+\mathcal{O}\left(G^{2}\right)$ and $\mathcal{S}_{i}^{\mu \nu}=$ 
$\frac{1}{2}\left(\mathcal{S}_{i,+\infty}^{\mu \nu}+\mathcal{S}_{i,-\infty}^{\mu \nu}\right)+\mathcal{O}\left(G^{2}\right)$. To leading order in $G$ the transition is straightforwardly accomplished using $\left\langle\Delta b^{\mu}\right\rangle$, the momentum impulse $\left\langle\Delta p_{i}^{\mu}\right\rangle=m_{i}\left(v_{i,+\infty}^{\mu}-v_{i,-\infty}^{\mu}\right)$ and the spin $\operatorname{kick}\left\langle\Delta S_{i}^{\mu \nu}\right\rangle=\mathcal{S}_{i,+\infty}^{\mu \nu}-\mathcal{S}_{i,-\infty}^{\mu \nu}$ :

$$
\begin{gathered}
b_{ \pm \infty}^{\mu}=b^{\mu} \pm \frac{\left\langle\Delta b^{\mu}\right\rangle}{2}+\mathcal{O}\left(G^{2}\right) \\
v_{i, \pm \infty}^{\mu}=v_{i}^{\mu} \pm \frac{\left\langle\Delta p_{i}^{\mu}\right\rangle}{2 m_{i}}+\mathcal{O}\left(G^{2}\right) \\
\mathcal{S}_{i, \pm \infty}^{\mu \nu}=\mathcal{S}_{i}^{\mu \nu} \pm \frac{\left\langle\Delta \mathcal{S}_{i}^{\mu \nu}\right\rangle}{2}+\mathcal{O}\left(G^{2}\right) .
\end{gathered}
$$

Using $0=v_{i}^{\mu}\left\langle\Delta p_{i, \mu}\right\rangle$ (4.34a) it also follows that $\gamma_{ \pm \infty}=\gamma+\mathcal{O}\left(G^{2}\right)$. Given our 1PM results in section 5.2 we lack only the $1 \mathrm{PM}$ expression for $\left\langle\Delta b^{\mu}\right\rangle$; those results are unaffected as the parameters differ by terms $\mathcal{O}(G)$.

Conservation of angular momentum at $1 \mathrm{PM}$ order $\left\langle\Delta J^{\mu \nu}\right\rangle=\mathcal{J}_{+\infty}^{\mu \nu}-\mathcal{J}_{-\infty}^{\mu \nu}=0$ gives us an $\mathcal{O}(G)$ expression for $\left\langle\Delta b^{\mu}\right\rangle$. The total angular momentum at future/past infinity is

$$
\begin{aligned}
\mathcal{J}_{ \pm \infty}^{\mu \nu} & =\sum_{i=1}^{2} m_{i}\left(2 b_{i, \pm \infty}^{[\mu} v_{i, \pm \infty}^{\nu]}+\mathcal{S}_{i, \pm \infty}^{\mu \nu}\right) \\
& =2\left(b_{1, \pm \infty}+b_{2, \pm \infty}\right)^{[\mu} P^{\nu]}+2 q_{ \pm \infty}^{[\mu} b_{ \pm \infty}^{\nu]}+\sum_{i=1}^{2} m_{i} \mathcal{S}_{i, \pm \infty}^{\mu \nu}
\end{aligned}
$$

where $P^{\mu}:=\frac{1}{2}\left(m_{1} v_{1, \pm \infty}^{\mu}+m_{2} v_{2, \pm \infty}^{\mu}\right)$ and $q_{ \pm \infty}^{\mu}:=\frac{1}{2}\left(m_{1} v_{1, \pm \infty}^{\mu}-m_{2} v_{2, \pm \infty}^{\mu}\right)$. We note that $\left\langle\Delta p_{1}^{\mu}\right\rangle=-\left\langle\Delta p_{2}^{\mu}\right\rangle=q_{+\infty}^{\mu}-q_{-\infty}^{\mu}$ due to conservation of linear momentum. To isolate the term depending on the relative impact parameter $b^{\mu}=b_{2}^{\mu}-b_{1}^{\mu}$ we introduce a projector to the $(D-1)$-dimensional space orthogonal to $P^{\mu}$ :

$$
\Lambda_{\nu}^{\mu}:=\delta_{\nu}^{\mu}-\frac{P^{\mu} P_{\nu}}{P^{2}} .
$$

In effect, contraction with this projector specializes us to the spacelike components of $\mathcal{J}_{ \pm \infty}^{\mu \nu}$ in the center-of-mass frame:

$$
\left(\Lambda \cdot \mathcal{J}_{ \pm \infty} \cdot \Lambda\right)^{\mu \nu}=2\left(\Lambda \cdot q_{ \pm \infty}\right)^{[\mu}\left(\Lambda \cdot b_{ \pm \infty}\right)^{\nu]}+\sum_{i=1}^{2} m_{i}\left(\Lambda \cdot \mathcal{S}_{i, \pm \infty} \cdot \Lambda\right)^{\mu \nu}
$$

Contracting $\Lambda^{\mu}{ }_{\rho} \Lambda^{\nu}{ }_{\sigma}\left\langle\Delta J^{\rho \sigma}\right\rangle=0$ with $q^{\mu}=\frac{1}{2}\left(m_{1} v_{1}^{\mu}-m_{2} v_{2}^{\mu}\right)$ we rearrange to find an $\mathcal{O}(G)$ expression for $\left\langle\Delta b^{\mu}\right\rangle$ :

$$
\left\langle\Delta b^{\mu}\right\rangle^{(1)}=\frac{\Lambda^{\mu \nu}}{q \cdot \Lambda \cdot q}\left(-q_{\nu} b^{\rho}\left\langle\Delta p_{1, \rho}\right\rangle^{(1)}+\sum_{i=1}^{2} m_{i}\left\langle\Delta S_{i, \nu \rho}\right\rangle^{(1)} \Lambda^{\rho \sigma} q_{\sigma}\right),
$$

having inserted the expressions for $b_{ \pm \infty}^{\mu}, v_{i, \pm \infty}^{\mu}$ and $\mathcal{S}_{i, \pm \infty}^{\mu \nu}$ (5.12). We have also used the requirement that $b_{ \pm \infty} \cdot v_{i, \pm \infty}=0$, which using eq. (5.12) implies $P_{\mu}\left\langle\Delta b^{\mu}\right\rangle=0$ and $q_{\mu}\left\langle\Delta b^{\mu}\right\rangle=-b_{\mu}\left\langle\Delta p_{1}^{\mu}\right\rangle$.

Our preference is to re-express observables in terms of background parameters taken in the far past $\tau \rightarrow-\infty$; the switch should be performed only after taking derivatives of the 
eikonal (5.5). The $2 \mathrm{PM}$ observables then pick up corrections from the $1 \mathrm{PM}$ observables in section 5.2. As the results are quite lengthy we provide them here only in $D=4$ dimensions up to linear order in spin; for the full $D$-dimensional quadratic-in-spin expressions we refer the reader to the accompanying supplementary material. Firstly, the momentum impulse:

$$
\begin{aligned}
& \left.\left\langle\Delta p_{1}^{\mu}\right\rangle^{(2)}\right|_{\mathcal{S}_{1}^{0} \mathcal{S}_{2}^{0}}=\frac{m_{1} m_{2}}{|b|^{2}}\left(\frac{3 \pi\left(5 \gamma^{2}-1\right)\left(m_{1}+m_{2}\right)}{4 \sqrt{\gamma^{2}-1}} \hat{b}^{\mu}\right. \\
& \left.\quad-2 \frac{\left(2 \gamma^{2}-1\right)^{2}}{\left(\gamma^{2}-1\right)^{2}}\left(\left(\gamma m_{1}+m_{2}\right) v_{1}^{\mu}-\left(\gamma m_{2}+m_{1}\right) v_{2}^{\mu}\right)\right) \\
& \left.\left\langle\Delta p_{1}^{\mu}\right\rangle^{(2)}\right|_{\mathcal{S}_{1}^{1} \mathcal{S}_{2}^{0}}=\frac{m_{1} m_{2}}{|b|^{2}}\left(\frac { \hat { b } \cdot \mathcal { S } _ { 1 } \cdot v _ { 2 } } { | b | } \left(-\frac{3 \pi \gamma\left(5 \gamma^{2}-3\right)}{4\left(\gamma^{2}-1\right)^{3 / 2}}\left(4 m_{1}+3 m_{2}\right) \hat{b}^{\mu}\right.\right. \\
& \left.+\frac{16 \gamma^{2}\left(2 \gamma^{2}-1\right) m_{1}+2 \gamma\left(12 \gamma^{2}-5\right) m_{2}}{\left(\gamma^{2}-1\right)^{2}} v_{1}^{\mu}-\frac{16 \gamma\left(2 \gamma^{2}-1\right) m_{1}+2\left(8 \gamma^{4}-1\right) m_{2}}{\left(\gamma^{2}-1\right)^{2}} v_{2}^{\mu}\right) \\
& \left.-\frac{2\left(4 \gamma^{2}-1\right) m_{2}+8 \gamma\left(2 \gamma^{2}-1\right) m_{1}}{\gamma^{2}-1} \frac{\left(\hat{b} \cdot \mathcal{S}_{1}\right)^{\mu}}{|b|}+\frac{\pi \gamma\left(5 \gamma^{2}-3\right)\left(4 m_{1}+3 m_{2}\right)}{4\left(\gamma^{2}-1\right)^{3 / 2}} \frac{\left(v_{2} \cdot \mathcal{S}_{1}\right)^{\mu}}{|b|}\right) .
\end{aligned}
$$

Here, and from this point on, the $-\infty$ subscripts on $\hat{b}_{-\infty}^{\mu}, v_{i,-\infty}^{\mu}$ and $\mathcal{S}_{i,-\infty}^{\mu \nu}$ should be considered implicit. The $\mathcal{O}\left(G^{2}\right)$ part of the spin kick is

$$
\begin{aligned}
& \left\langle\Delta S_{1}^{\mu \nu}\right\rangle^{(2)}=\frac{m_{2}^{2}}{|b|^{2}\left(\gamma^{2}-1\right)}\left(4\left(\hat{b} \cdot \mathcal{S}_{1}\right)^{[\mu} \hat{b}^{\nu]}-16 \gamma \hat{b} \cdot \mathcal{S}_{1} \cdot v_{2}\left(2 \gamma v_{2}-v_{1}\right)^{[\mu} \hat{b}^{\nu]}\right. \\
& -16 \gamma^{2}\left(v_{2} \cdot \mathcal{S}_{1}\right)^{[\mu} v_{2}{ }^{\nu]}+\frac{\pi \gamma\left(5 \gamma^{2}-3\right)\left(4 m_{1}+3 m_{2}\right)}{2 \sqrt{\gamma^{2}-1} m_{2}}\left(\left(\hat{b} \cdot \mathcal{S}_{1}\right)^{[\mu} v_{2}{ }^{\nu]}-\left(v_{2} \cdot \mathcal{S}_{1}\right)^{[\mu} \hat{b}^{\nu]}\right) \\
& -\frac{\pi\left(5 \gamma^{4}+6 \gamma^{2}-3\right) m_{1}+3 \pi\left(3 \gamma^{2}-1\right) m_{2}}{2 \sqrt{\gamma^{2}-1} m_{2}}\left(\hat{b} \cdot \mathcal{S}_{1}\right)^{[\mu} v_{1}{ }^{\nu]} \\
& \left.+\frac{4\left(2 \gamma^{2}-1\right)^{2} m_{1}+4 \gamma\left(4 \gamma^{2}-3\right) m_{2}}{\left(\gamma^{2}-1\right) m_{2}}\left(v_{2} \cdot \mathcal{S}_{1}\right)^{[\mu} v_{1}{ }^{\nu]}\right)
\end{aligned}
$$

When expressed in terms of the new background parameters, different relationships are satisfied by these observables:

$$
\begin{aligned}
m_{i}^{2} v_{i}^{2} & =\left(m_{i} v_{i}^{\mu}+\left\langle\Delta p_{i}^{\mu}\right\rangle\right)^{2}, \\
\left(\mathcal{S}_{i}^{\mu \nu}\right)^{2} & =\left(\mathcal{S}_{i}^{\mu \nu}+\left\langle\Delta S_{i}^{\mu \nu}\right\rangle\right)^{2}, \\
m_{i} v_{i, \mu} \mathcal{S}_{i}^{\mu \nu} & =\left(m_{i} v_{i, \mu}+\left\langle\Delta p_{i, \mu}\right\rangle\right)\left(\mathcal{S}_{i}^{\mu \nu}+\left\langle\Delta S_{i}^{\mu \nu}\right\rangle\right) .
\end{aligned}
$$

However, the interpretation is still the same: supercharges are conserved between initial 
and final states. Lastly, the $2 \mathrm{PM}$ scattering angle in $D=4$ is

$$
\begin{aligned}
& \theta^{(2)}= \frac{E\left(m_{1}+m_{2}\right)}{|b|^{2}}\left(\frac{3 \pi\left(5 \gamma^{2}-1\right)}{4\left(\gamma^{2}-1\right)}+\frac{\pi \gamma\left(5 \gamma^{2}-3\right)}{2\left(\gamma^{2}-1\right)^{3 / 2}}\left(\frac{3 m_{2}+4 m_{1}}{m_{1}+m_{2}} \frac{s_{1}}{|b|}+(1 \leftrightarrow 2)\right)\right. \\
&+\frac{3 \pi}{2\left(\gamma^{2}-1\right)^{2}|b|^{2}}\left[\frac{\left(95 \gamma^{4}-102 \gamma^{2}+15\right) m_{1}+4\left(15 \gamma^{4}-15 \gamma^{2}+2\right) m_{2}}{8\left(m_{1}+m_{2}\right)} s_{1}^{2}+(1 \leftrightarrow 2)\right. \\
& \quad-C_{E, 1} \frac{\left(125 \gamma^{4}-138 \gamma^{2}+29\right) m_{1}+2\left(45 \gamma^{4}-42 \gamma^{2}+5\right) m_{2}}{16\left(m_{1}+m_{2}\right)} s_{1}^{2}+(1 \leftrightarrow 2) \\
&\left.\left.+\left(20 \gamma^{4}-21 \gamma^{2}+3\right) s_{1} s_{2}\right]\right)
\end{aligned}
$$

where the specialization to aligned spins was given earlier (5.10). The spin-free part of our 2PM scattering angle in $D$ dimensions (provided in the supplementary material) agrees with earlier results $[110,111]$.

Finally, we have observed that the $2 \mathrm{PM}$ eikonal phase $\chi$ presented in section 5.1 is invariant under the transformations between intermediate and past background parameters (5.12) - unlike the momentum impulse $\left\langle\Delta p_{1}^{\mu}\right\rangle$ and spin kick $\left\langle\Delta S_{1}^{\mu \nu}\right\rangle$ presented above. One may therefore freely replace $b^{\mu} \rightarrow b_{ \pm \infty}^{\mu}, v_{i}^{\mu} \rightarrow v_{i, \pm \infty}^{\mu}$ and $\mathcal{S}_{i}^{\mu \nu} \rightarrow \mathcal{S}_{i, \pm \infty}^{\mu \nu}$ in the expressions (5.3) and (5.4) without changing their validity. However, one should then exercise caution when deriving physical observables: the simple derivatives of the eikonal phase (5.5) apply only to the "averaged" background parameters, rather than those defined at past/future infinity (5.12). Instead, following the approach conjectured in refs. [55, 62], it is appropriate to include terms quadratic in the eikonal phase. Noting that the shifts (5.12) cannot be considered instances of the background field symmetries discussed in section 4.4 (e.g. the background symmetries (4.32) leave $v_{i}^{\mu}$ invariant) the additional invariance here suggests the existence of a larger class of conserved quantities than those limited here to the individual worldlines. We leave this tantalizing question for future work.

\section{Conclusions}

The $\mathcal{N}=2$ supersymmetric worldline action provides an alternative description of a compact spinning object up to terms quadratic in spin $\mathcal{O}\left(\mathcal{S}^{2}\right)$ (quadrupoles). Using this equivalence we have shown how quadratic-in-spin effects may be incorporated into the worldline quantum field theory (WQFT) prescription for scattering massive bodies in a curved background [1]. The classical spin tensors $S_{i}^{a b}=-2 i \bar{\psi}_{i}^{[a} \psi_{i}^{b]}$ (in a local frame $e_{a}^{\mu}$ ) are considered composite fields, built from the complex Grassmann-valued vectors $\psi_{i}^{a}$ living on each worldline $i$. Conveniently, this provides for a Lagrangian worldline formalism that involves neither a body-fixed frame nor angular velocity tensor. The technology was previously used to obtain the far-field time-domain waveform from a scattering of two massive bodies (black holes, neutron stars or stars) to leading order in $G[3]$; here we elaborated on it further.

While Kerr black holes are privileged, and represented by the unique $\mathcal{N}=2$ supersymmetric theory, finite-size effects may also be incorporated starting at $\mathcal{O}\left(\mathcal{S}^{2}\right)$ by adding terms that only preserve SUSY approximately (up to $\mathcal{O}\left(\psi^{5}\right)$ ). The conserved supercharges 
have natural physical interpretations: conservation of energy, spin length and the spinsupplementary condition (SSC) $p_{\mu} S^{\mu \nu}=0$ along each worldline. While these are conserved locally in the supersymmetric theory, with the inclusion of finite-size effects they are only conserved approximately up to $\mathcal{O}\left(\mathcal{S}^{3}\right)$. The analogue can be seen in ref. [3], where the time-domain waveform is approximately supersymmetric when finite-size effects are included and exactly supersymmetric in the Kerr-black hole case.

Our main result is an explicit expression for the $D$-dimensional eikonal phase $\chi=$ $-i \log \mathcal{Z}_{\mathrm{WQFT}}$ up to $\mathcal{O}\left(G^{2}\right)$ (2PM order), where $\mathcal{Z}_{\mathrm{WQFT}}$ is the partition function of the WQFT. This was obtained as a sum of tree-level vacuum diagrams integrated over the momenta (or energies on the worldlines) of internal lines. From the eikonal phase we showed how one may derive three key observables: the momentum impulse $\left\langle\Delta p_{1}^{\mu}\right\rangle=-\left\langle\Delta p_{2}^{\mu}\right\rangle$, spin kicks $\left\langle\Delta S_{i}^{\mu \nu}\right\rangle$ and (for aligned spins) the scattering angle $\theta$. In $D=4$ dimensions these observables agree with previous results [30,62]. The requirements of energy conservation and preservation of both the spin-supplementary condition (SSC) and spin length follow naturally from the supersymmetry. Another important subtlety is the interpretation of background parameters $b_{i}^{\mu}, v_{i}^{\mu}$ and $\mathcal{S}_{i}^{\mu \nu}$ : in the eikonal phase these are defined at an intermediate point of the scattering, and an interpolation is needed to relate them to those in the far past $(\tau \rightarrow-\infty)$.

Our work offers numerous follow-up opportunities, which we will explore enthusiastically. Naturally, one wonders about the prospects for extending the formalism beyond quadratic order in the spins. As explained in section 2.1, in a flat spacetime background there exist $\mathcal{N}$-supersymmetric worldline theories with real Grassmann-valued vectors $\psi_{\alpha}^{a}(\tau)$ carrying flavor indices $\alpha=1, \ldots, \mathcal{N}$ that generically describe the propagation of spin- $\mathcal{N} / 2$ particles $[78,103,104]$. The main obstacle is generalizing these theories to an arbitrary curved spacetime background whilst preserving supersymmetry. Yet we have seen that perturbative deformations of the supercharges yielding an approximate supersymmetry are possible; it would be worthwhile to revisit the issue under these premises. Fortunately, the higher spin limitation does not exist in gauge theories: the so-called $\sqrt{\text { Kerr }}$ theory [112], which enjoys a complex worldsheet description [102], is a natural candidate for study. Given ongoing research on the double copy in WQFT [113], this could provide a window on higher spins for the Kerr black hole and is left for future work.

We also see excellent prospects for applying the spinning WQFT formalism to higherPM order calculations. As explained in ref. [1] we are not limited to using the eikonal phase: we can also compute $\left\langle\Delta p_{i}^{\mu}\right\rangle$ directly by drawing graphs with an outgoing deflection mode $z_{i}^{\mu}$; similarly we can obtain $\left\langle\Delta \psi_{i}^{\mu}\right\rangle$, and therefore $\left\langle\Delta S_{i}^{\mu \nu}\right\rangle$ via eq. (4.29), by drawing graphs with an outgoing $\psi_{i}^{\prime \mu}$ line. There already has been excellent progress at 3PM order in the non-spinning case $[39,71,75,114]$ including radiation reaction effects $[41,60]$.

Finally, in the non-spinning case a link between scalar-graviton S-matrix elements and operator expectation values in the WQFT has been formally provided by a worldline path integral "Feynman-Schwinger" representation of the graviton-dressed scalar propagator [1]. We would like to extend this link to include spin effects - again, gauge theory will provide a useful starting point given that the $n$-dressed electron propagator is already known [115, 116] (see refs. [117-119] for comprehensive reviews). In gravity, such a dressed propagator 
is not currently known, and when obtained will provide for a complete theoretical map between the different PM-based approaches to spinning black hole scattering.

\section{Acknowledgments}

We would like to thank Roberto Bonezzi, Alessandra Buonanno, Paolo Pichini, Christian Schubert and Justin Vines for helpful discussions. We are also grateful for use of Gregor Kälin's C++ graph library. GUJ's and GM's research is funded by the Deutsche Forschungsgemeinschaft (DFG, German Research Foundation), Projektnummer 417533893/GRK2575 "Rethinking Quantum Field Theory".

\section{A Supersymmetry}

The relevant part of the $e=1 / m$ gauge-fixed $\mathcal{N}=2$ worldline action in curved space $(2.16)$ reads

$$
S=-m \int \mathrm{d} \tau\left[\frac{1}{2} g_{\mu \nu} \dot{x}^{\mu} \dot{x}^{\nu}+i \bar{\psi}^{a} \dot{\psi}_{a}+i \dot{x}^{\mu} \omega_{\mu a b} \bar{\psi}^{a} \psi^{b}+\frac{1}{2} R_{a b c d} \bar{\psi}^{a} \psi^{b} \bar{\psi}^{c} \psi^{d}\right] .
$$

and we now want to prove its SUSY invariance. The SUSY transformations of $x^{\mu}, \psi^{a}$ and $\bar{\psi}^{a}$ were quoted in eq. (2.19):

$$
\delta x^{\mu}=i e_{a}^{\mu}\left(\bar{\epsilon} \psi^{a}+\epsilon \bar{\psi}^{a}\right), \quad \delta \psi^{a}=-\epsilon e_{\mu}^{a} \dot{x}^{\mu}-\delta x^{\mu} \omega_{\mu}^{a} b \psi^{b},
$$

and are augmented by

$$
\delta e_{\mu}^{a}=\partial_{\nu} e_{\mu}^{a} \delta x^{\nu}, \quad \delta \omega_{\mu a b}=\partial_{\nu} \omega_{\mu a b} \delta x^{\nu}, \quad \delta R_{a b c d}=\partial_{\nu} R_{a b c d} \delta x^{\nu} .
$$

In order to show the invariance of the action $S$ we analyze the variation $\delta S$ order-by-order in the fermions $\psi^{a}$. At linear order only the variations of the first three terms in eq. (A.1) contribute and one finds

$$
\left.\delta S\right|_{\text {lin }}=-m \int d \tau\left(g_{\mu \nu} \dot{x}^{\mu} i \bar{\epsilon} \dot{\psi}^{\nu}-\dot{x}^{\mu} e_{\mu}^{a} i \bar{\epsilon} \dot{\psi}^{a}+\left[\frac{1}{2} e_{a}^{\rho} \partial_{\rho} g_{\mu \nu}-\omega_{(\mu \nu) a}\right] i \bar{\epsilon} \psi^{a} \dot{x}^{\mu} \dot{x}^{\nu}+\text { c.c }\right) .
$$

Using $\frac{1}{2} e_{a}^{\rho} \partial_{\rho} g_{\mu \nu}-\omega_{(\mu \nu) a}=-g_{\rho(\mu} \partial_{\nu)} e_{a}^{\rho}$ the last term is rewritten as $-g_{\rho \mu} \dot{x}^{\mu}\left(\frac{d}{d \tau} e_{a}^{\rho}\right) i \bar{\epsilon} \psi^{a}$. Noting that the first two terms combine to the same expression, but an opposite sign, we see the vanishing of the linear in $\psi^{a}$ variation $\left.\delta S\right|_{\text {lin }}$. At cubic order one picks up contributions only from the last three terms in eq. (A.1) and finds

$$
\left.\delta S\right|_{\text {cubic }}=-m \int d \tau\left[\partial_{\rho} \omega_{\mu a b}-\partial_{\mu} \omega_{\rho a b}-\omega_{\mu a c} \omega_{\rho b}^{c}+\omega_{\rho a c} \omega_{\mu}^{c} b+R_{\mu \rho a b}\right] i \dot{x}^{\mu} \delta x^{\rho} \bar{\psi}^{a} \psi^{b},
$$

which vanishes identically using the spin-connection based definition of the Riemann-tensor. At quintic order one merely considers the variation of the last term in eq. (A.1). Here the three types of contributions conspire to yield

$$
\begin{aligned}
& \left.\delta S\right|_{\text {quintic }}= \\
& \quad=-m \int d \tau\left[\partial_{\mu} R_{a b c d}+\omega_{\mu a}^{e} R_{e b c d}+\omega_{\mu b}^{e} R_{a e c d}+\omega_{\mu c}^{e} R_{a b e d}+\omega_{\mu d}^{e} R_{a b c e}\right] \delta x^{\mu} \bar{\psi}^{a} \psi^{b} \bar{\psi}^{c} \psi^{d},
\end{aligned}
$$


which constitutes the covariant derivative of the Riemann-tensor $\nabla_{\mu} R_{a b c d}$. By virtue of $\nabla_{\mu} e_{\nu}^{a}=0$ we need to show the vanishing of the term $\nabla_{\mu} R_{\alpha \nu \beta \rho} \psi^{\mu} \psi^{\nu} \psi^{\rho}$ (and analogously for $\psi \rightarrow \bar{\psi}$ ). Using the cyclicity of the Riemann tensor in its last three indices, $R_{\alpha \nu \beta \rho}=$ $-R_{\alpha \beta \rho \nu}-R_{\alpha \rho \nu \beta}$, and the anti-commuativity of the $\psi$ 's we have

$$
\nabla_{\mu} R_{\alpha \nu \beta \rho} \psi^{\mu} \psi^{\nu} \psi^{\rho}=\frac{1}{2} \nabla_{\mu} R_{\alpha \beta \nu \rho} \psi^{\mu} \psi^{\nu} \psi^{\rho} .
$$

This expression vanishes by virtue of the Biancchi identity $\nabla_{\mu} R_{\alpha \beta \nu \rho}+\nabla_{\nu} R_{\alpha \beta \rho \mu}+$ $\nabla_{\rho} R_{\alpha \beta \mu \nu}=0$.

For completeness, let us now also look at the supersymmetry variation of the finite-size term (4.2) relevant for (neutron) stars:

$$
S_{E}=-m C_{E} \int \mathrm{d} \tau R_{a \mu b \nu} \dot{x}^{\mu} \dot{x}^{\nu} \bar{\psi}^{a} \psi^{b} P_{c d} \bar{\psi}^{c} \psi^{d} .
$$

Varying this under (A.2) we produce terms of order three and five in the worldline fermions $\psi^{a}$. The order-five terms would also receive contributions from putative order-six terms (yielding $\operatorname{spin}^{3}$ effects) in the effective worldline theory that we have not considered. Hence, of relevance here are only the order-three terms in the supersymmetry variation of (A.8), which in fact come close to vanishing: varying the first two fermions in $S_{E}$ yields zero due to $R_{\rho \mu b \nu} \dot{x}^{\mu} \dot{x}^{\nu} \dot{x}^{\rho}=0$. One is left with

$$
\left.\delta S_{E}\right|_{\psi^{3}}=m C_{E} \int \mathrm{d} \tau R_{a \mu b \nu} \dot{x}^{\mu} \dot{x}^{\nu} \bar{\psi}^{a} \psi^{b} g_{\rho \sigma}\left(\bar{\epsilon} P_{\rho \sigma} \psi^{\rho} \dot{x}^{\sigma}+\epsilon P_{\rho \sigma} \bar{\psi}^{\rho} \dot{x}^{\sigma}\right) .
$$

The terms in the bracket vanish by virtue of the projector property: $P_{\rho \sigma} \dot{x}^{\sigma}=0$. Hence the finite-size term $S_{E}$ is supersymmetric approximately, i.e.

$$
\delta S_{E}=\mathcal{O}\left(\psi^{5}\right)
$$

As we would need to include a new layer of $\psi^{6}$ terms in order to describe spinning massive objects at the spin-cubed order, the SUSY variation of these not-considered terms would induce $\mathcal{O}\left(\psi^{5}\right)$ terms which would talk to the above.

\section{B Integrals}

To integrate the $1 \mathrm{PM}$ contribution to the eikonal phase $\chi$ given in eq. (5.2) we require expressions for the following class of $D$-dimensional Fourier transforms:

$$
I_{\nu}^{\mu_{1} \mu_{2} \ldots \mu_{n}}(D):=\int_{q} e^{i q \cdot b} \delta\left(q \cdot v_{1}\right) \delta\left(q \cdot v_{2}\right)|q|^{\nu} q^{\mu_{1}} q^{\mu_{2}} \cdots q^{\mu_{n}}
$$

where $|q|^{2}=-q \cdot q$ and $n \leq 2 ;^{8}$ the $\nu \neq-1$ generalization becomes relevant at 2PM order. The scalar integral is straightforwardly evaluated in a $(D-2)$-dimensional space orthogonal to $v_{1}^{\mu}$ and $v_{2}^{\mu}$ (see e.g. ref. [1]) with the well-known result:

$$
I_{\nu}(D)=\frac{2^{\nu}}{\pi^{(D-2) / 2} \sqrt{\gamma^{2}-1}} \frac{\Gamma\left(\frac{D-2+\nu}{2}\right)}{\Gamma\left(-\frac{\nu}{2}\right)}\left(-b \cdot P_{12} \cdot b\right)^{-\frac{D-2+\nu}{2}},
$$

\footnotetext{
${ }^{8} \mathrm{An} \mathcal{O}\left(\mathcal{S}^{\alpha}\right)$ contribution generically requires integrals with rank $n=\alpha$.
} 
where the projector $P_{12}^{\mu \nu}$ to the $(D-2)$-dimensional space orthogonal to $v_{i}^{\mu}$ was given in eq. (5.7). The generalization to higher-rank integrals follows easily by taking derivatives with respect to $b^{\mu}$ :

$$
I_{\nu}^{\mu_{1} \mu_{2} \ldots \mu_{n}}(D)=(-i)^{n} \frac{\partial^{n}}{\partial b_{\left(\mu_{1}\right.} \partial b_{\mu_{2}} \cdots \partial b_{\left.\mu_{n}\right)}} I_{\nu}(D) .
$$

It is important not to impose $b \cdot v_{i}=0$ until after these derivatives have been taken hence our use of the projector $P_{12}^{\mu \nu}$.

To integrate the various contributions appearing at $2 \mathrm{PM}$ order we additionally require full knowledge of the following family of integrals:

$$
J_{\nu_{1}, \nu_{2}, \nu_{3}}^{\mu_{1} \mu_{2} \ldots \mu_{n}}(D):=\int_{\ell} \frac{\delta\left(\ell \cdot v_{1}\right)}{\left(\ell^{2}+i \epsilon\right)^{\nu_{1}}\left((\ell-q)^{2}+i \epsilon\right)^{\nu_{2}}\left(\ell \cdot v_{2}+i \epsilon\right)^{\nu_{3}}} \ell^{\mu_{1}} \ell^{\mu_{2}} \cdots \ell^{\mu_{n}}
$$

with $v_{1}^{\mu} \leftrightarrow v_{2}^{\mu}$ related by symmetry and $n \leq 3$. The scalar integral is straightforwardly evaluated by choosing the rest frame of the first body, $v_{1}^{\mu}=(1, \mathbf{0})$, and performing the resulting $(D-1)$-dimensional one-loop integral:

$$
J_{\nu_{1}, \nu_{2}, \nu_{3}}(D)=(-i)^{2 \nu_{1}+2 \nu_{2}+\nu_{3}}(4 \pi)^{\frac{1-D}{2}}\left(\frac{4}{\gamma^{2}-1}\right)^{\frac{\nu_{3}}{2}} \Gamma_{\nu_{1}, \nu_{2}, \nu_{3}}(D-1)|q|^{D-1-2 \nu_{1}-2 \nu_{2}-\nu_{3}},
$$

where

$$
\Gamma_{\nu_{1}, \nu_{2}, \nu_{3}}(D):=\frac{\Gamma\left(\nu_{1}+\nu_{2}+\frac{\nu_{3}}{2}-\frac{D}{2}\right) \Gamma\left(\frac{\nu_{3}}{2}\right)}{2 \Gamma\left(\nu_{1}\right) \Gamma\left(\nu_{2}\right) \Gamma\left(\nu_{3}\right)} \frac{\Gamma\left(\frac{D}{2}-\nu_{1}-\frac{\nu_{3}}{2}\right) \Gamma\left(\frac{D}{2}-\nu_{2}-\frac{\nu_{3}}{2}\right)}{\Gamma\left(D-\nu_{1}-\nu_{2}-\nu_{3}\right)} .
$$

When $\nu_{3}=0$ we use $\Gamma\left(\frac{\nu_{3}}{2}\right)=2 \Gamma\left(\nu_{3}\right) \cdot{ }^{9}$ Higher-rank integrals are expanded on a basis of tensors living in the $(D-1)$-dimensional space orthogonal to $v_{1}^{\mu}$ : namely $P_{1}^{\mu \nu}, P_{1}^{\mu \nu} v_{2, \nu}$, and $q^{\mu}$, where $P_{1}^{\mu \nu}:=\eta^{\mu \nu}-v_{1}^{\mu} v_{1}^{\nu}$ is the projector orthogonal to $v_{1}^{\mu}$. The coefficients are found by contracting an ansatz with these tensors, and for example with $n=1$ one can easily show that

$$
J_{\nu_{1}, \nu_{2}, \nu_{3}}^{\mu}=\frac{q^{\mu}}{2|q|^{2}}\left(|q|^{2} J_{\nu_{1}, \nu_{2}, \nu_{3}}-J_{\nu_{1}-1, \nu_{2}, \nu_{3}}+J_{\nu_{1}, \nu_{2}-1, \nu_{3}}\right)+\frac{P_{1}^{\mu \nu} v_{2, \nu}}{\gamma^{2}-1} J_{\nu_{1}, \nu_{2}, \nu_{3}-1},
$$

which holds in any dimension $D$. Similar relations hold for $n=2$ and $n=3$.

Open Access. This article is distributed under the terms of the Creative Commons Attribution License (CC-BY 4.0), which permits any use, distribution and reproduction in any medium, provided the original author(s) and source are credited.

\section{References}

[1] G. Mogull, J. Plefka and J. Steinhoff, Classical black hole scattering from a worldline quantum field theory, JHEP 02 (2021) 048 [arXiv: 2010.02865] [INSPIRE].

[2] G.U. Jakobsen, G. Mogull, J. Plefka and J. Steinhoff, Classical gravitational bremsstrahlung from a worldline quantum field theory, Phys. Rev. Lett. 126 (2021) 201103 [arXiv:2101.12688] [INSPIRE].

\footnotetext{
${ }^{9}$ As internal self-consistency checks we also found it helpful to consider integration-by-parts (IBP) relations between these integrals using LiteRed [120, 121] and FIRE6 [122].
} 
[3] G.U. Jakobsen, G. Mogull, J. Plefka and J. Steinhoff, Gravitational bremsstrahlung and hidden supersymmetry of spinning bodies, Phys. Rev. Lett. 128 (2022) 011101 [arXiv:2106.10256] [INSPIRE].

[4] G.W. Gibbons, R.H. Rietdijk and J.W. van Holten, SUSY in the sky, Nucl. Phys. B 404 (1993) 42 [hep-th/9303112] [INSPIRE].

[5] K.S. Thorne and S.J. Kovacs, The generation of gravitational waves. I. Weak-field sources, Astrophys. J. 200 (1975) 245.

[6] R.J. Crowley and K.S. Thorne, The generation of gravitational waves. II. The postlinear formalism revisited, Astrophys. J. 215 (1977) 624 [INSPIRE].

[7] S.J. Kovacs and K.S. Thorne, The generation of gravitational waves. III. Derivation of bremsstrahlung formulas, Astrophys. J. 217 (1977) 252 [INSPIRE].

[8] S.J. Kovacs and K.S. Thorne, The generation of gravitational waves. IV. Bremsstrahlung, Astrophys. J. 224 (1978) 62 [INSPIRE].

[9] L. Bel, T. Damour, N. Deruelle, J. Ibáñez and J. Martin, Poincaré-invariant gravitational field and equations of motion of two pointlike objects: the postlinear approximation of general relativity, Gen. Rel. Grav. 13 (1981) 963 [INSPIRE].

[10] K. Westpfahl, High-speed scattering of charged and uncharged particles in general relativity, Fortsch. Phys. 33 (1985) 417 [InSPIRE].

[11] LIGO ScIEnTIFIC and VIRGo collaborations, Observation of gravitational waves from a binary black hole merger, Phys. Rev. Lett. 116 (2016) 061102 [arXiv:1602.03837] [INSPIRE].

[12] LIGO SCIENTIFIC and VIRGO collaborations, GWTC-1: a gravitational-wave transient catalog of compact binary mergers observed by LIGO and Virgo during the first and second observing runs, Phys. Rev. X 9 (2019) 031040 [arXiv:1811.12907] [INSPIRE].

[13] LIGO SCIENTIFIC and VIRGO collaborations, GWTC-2: compact binary coalescences observed by LIGO and Virgo during the first half of the third observing run, Phys. Rev. $X$ 11 (2021) 021053 [arXiv: 2010.14527] [INSPIRE].

[14] LIGO ScIENTIFIC and VIRGO collaborations, GWTC-2.1: deep extended catalog of compact binary coalescences observed by LIGO and Virgo during the first half of the third observing run, arXiv:2108.01045 [INSPIRE].

[15] B. Kocsis, M.E. Gaspar and S. Marka, Detection rate estimates of gravity-waves emitted during parabolic encounters of stellar black holes in globular clusters, Astrophys. J. 648 (2006) 411 [astro-ph/0603441] [INSPIRE].

[16] S. Mukherjee, S. Mitra and S. Chatterjee, Gravitational wave observatories may be able to detect hyperbolic encounters of black holes, Mon. Not. Roy. Astron. Soc. 508 (2021) 5064 [arXiv:2010.00916] [INSPIRE].

[17] M. Zevin, J. Samsing, C. Rodriguez, C.-J. Haster and E. Ramirez-Ruiz, Eccentric black hole mergers in dense star clusters: the role of binary-binary encounters, Astrophys. J. 871 (2019) 91 [arXiv:1810.00901] [INSPIRE].

[18] C. Cheung, I.Z. Rothstein and M.P. Solon, From scattering amplitudes to classical potentials in the post-Minkowskian expansion, Phys. Rev. Lett. 121 (2018) 251101 [arXiv: 1808.02489] [INSPIRE]. 
[19] D. Neill and I.Z. Rothstein, Classical space-times from the $S$ matrix, Nucl. Phys. B 877 (2013) 177 [arXiv: 1304.7263] [INSPIRE].

[20] V. Vaidya, Gravitational spin Hamiltonians from the S matrix, Phys. Rev. D 91 (2015) 024017 [arXiv: 1410.5348] [INSPIRE].

[21] T. Damour, High-energy gravitational scattering and the general relativistic two-body problem, Phys. Rev. D 97 (2018) 044038 [arXiv:1710.10599] [INSPIRE].

[22] N.E.J. Bjerrum-Bohr, A. Cristofoli and P.H. Damgaard, Post-Minkowskian scattering angle in Einstein gravity, JHEP 08 (2020) 038 [arXiv:1910.09366] [INSPIRE].

[23] G. Kälin and R.A. Porto, From boundary data to bound states, JHEP 01 (2020) 072 [arXiv: 1910.03008] [INSPIRE].

[24] G. Kälin and R.A. Porto, From boundary data to bound states. Part II. Scattering angle to dynamical invariants (with twist), JHEP 02 (2020) 120 [arXiv: 1911.09130] [INSPIRE].

[25] W.D. Goldberger and I.Z. Rothstein, An effective field theory of gravity for extended objects, Phys. Rev. D 73 (2006) 104029 [hep-th/0409156] [INSPIRE].

[26] W.D. Goldberger and I.Z. Rothstein, Towers of gravitational theories, Gen. Rel. Grav. 38 (2006) 1537 [Int. J. Mod. Phys. D 15 (2006) 2293] [hep-th/0605238] [INSPIRE].

[27] W.D. Goldberger and A. Ross, Gravitational radiative corrections from effective field theory, Phys. Rev. D 81 (2010) 124015 [arXiv:0912.4254] [INSPIRE].

[28] R.A. Porto, Post-Newtonian corrections to the motion of spinning bodies in NRGR, Phys. Rev. D 73 (2006) 104031 [gr-qc/0511061] [INSPIRE].

[29] M. Levi and J. Steinhoff, Spinning gravitating objects in the effective field theory in the post-Newtonian scheme, JHEP 09 (2015) 219 [arXiv:1501.04956] [INSPIRE].

[30] Z. Liu, R.A. Porto and Z. Yang, Spin effects in the effective field theory approach to post-Minkowskian conservative dynamics, JHEP 06 (2021) 012 [arXiv:2102.10059] [INSPIRE].

[31] G. Kälin, Z. Liu and R.A. Porto, Conservative tidal effects in compact binary systems to next-to-leading post-Minkowskian order, Phys. Rev. D 102 (2020) 124025 [arXiv:2008.06047] [INSPIRE].

[32] J. Blümlein, A. Maier, P. Marquard and G. Schäfer, Testing binary dynamics in gravity at the sixth post-Newtonian level, Phys. Lett. B 807 (2020) 135496 [arXiv:2003.07145] [INSPIRE].

[33] D. Bini, T. Damour and A. Geralico, Sixth post-Newtonian local-in-time dynamics of binary systems, Phys. Rev. D 102 (2020) 024061 [arXiv:2004.05407] [InSPIRE].

[34] D. Bini, T. Damour and A. Geralico, Sixth post-Newtonian nonlocal-in-time dynamics of binary systems, Phys. Rev. D 102 (2020) 084047 [arXiv:2007.11239] [INSPIRE].

[35] J. Blümlein, A. Maier, P. Marquard and G. Schäfer, The fifth-order post-Newtonian Hamiltonian dynamics of two-body systems from an effective field theory approach: potential contributions, Nucl. Phys. B 965 (2021) 115352 [arXiv:2010.13672] [INSPIRE].

[36] S. Foffa, R. Sturani and W.J. Torres Bobadilla, Efficient resummation of high post-Newtonian contributions to the binding energy, JHEP 02 (2021) 165 [arXiv:2010.13730] [INSPIRE]. 
[37] J. Blümlein, A. Maier, P. Marquard and G. Schäfer, The $6^{\text {th }}$ post-Newtonian potential terms at $O\left(G_{N}^{4}\right)$, Phys. Lett. B 816 (2021) 136260 [arXiv:2101.08630] [inSPIRE].

[38] G. Kälin and R.A. Porto, Post-Minkowskian effective field theory for conservative binary dynamics, JHEP 11 (2020) 106 [arXiv:2006.01184] [INSPIRE].

[39] G. Kälin, Z. Liu and R.A. Porto, Conservative dynamics of binary systems to third post-Minkowskian order from the effective field theory approach, Phys. Rev. Lett. 125 (2020) 261103 [arXiv: 2007.04977] [INSPIRE].

[40] C. Dlapa, G. Kälin, Z. Liu and R.A. Porto, Dynamics of binary systems to fourth post-Minkowskian order from the effective field theory approach, arXiv:2106.08276 [INSPIRE].

[41] D. Bini, T. Damour and A. Geralico, Radiative contributions to gravitational scattering, Phys. Rev. D 104 (2021) 084031 [arXiv:2107.08896] [InSPIRE].

[42] N.E.J. Bjerrum-Bohr, J.F. Donoghue and P. Vanhove, On-shell techniques and universal results in quantum gravity, JHEP 02 (2014) 111 [arXiv:1309.0804] [INSPIRE].

[43] N.E.J. Bjerrum-Bohr, P.H. Damgaard, G. Festuccia, L. Planté and P. Vanhove, General relativity from scattering amplitudes, Phys. Rev. Lett. 121 (2018) 171601 [arXiv: 1806. 04920] [INSPIRE].

[44] Z. Bern, C. Cheung, R. Roiban, C.-H. Shen, M.P. Solon and M. Zeng, Scattering amplitudes and the conservative Hamiltonian for binary systems at third post-Minkowskian order, Phys. Rev. Lett. 122 (2019) 201603 [arXiv: 1901.04424] [INSPIRE].

[45] Z. Bern, C. Cheung, R. Roiban, C.-H. Shen, M.P. Solon and M. Zeng, Black hole binary dynamics from the double copy and effective theory, JHEP 10 (2019) 206 [arXiv: 1908.01493] [INSPIRE].

[46] C. Cheung and M.P. Solon, Classical gravitational scattering at $\mathcal{O}\left(G^{3}\right)$ from Feynman diagrams, JHEP 06 (2020) 144 [arXiv:2003.08351] [INSPIRE].

[47] A. Luna, I. Nicholson, D. O'Connell and C.D. White, Inelastic black hole scattering from charged scalar amplitudes, JHEP 03 (2018) 044 [arXiv:1711.03901] [INSPIRE].

[48] L.J. Dixon, Calculating scattering amplitudes efficiently, in Theoretical Advanced Study Institute in elementary particle physics (TASI 95): QCD and beyond, (1996), pg. 539 [hep-ph/9601359] [INSPIRE].

[49] H. Elvang and Y.-T. Huang, Scattering amplitudes, arXiv:1308.1697 [INSPIRE].

[50] J.M. Henn and J.C. Plefka, Scattering amplitudes in gauge theories, Lect. Notes Phys. 883 (2014) 1 [INSPIRE].

[51] Z. Bern, J.J. Carrasco, M. Chiodaroli, H. Johansson and R. Roiban, The duality between color and kinematics and its applications, arXiv:1909.01358 [INSPIRE].

[52] D.A. Kosower, B. Maybee and D. O'Connell, Amplitudes, observables, and classical scattering, JHEP 02 (2019) 137 [arXiv:1811.10950] [INSPIRE].

[53] B. Maybee, D. O'Connell and J. Vines, Observables and amplitudes for spinning particles and black holes, JHEP 12 (2019) 156 [arXiv:1906.09260] [INSPIRE].

[54] T. Damour, Classical and quantum scattering in post-Minkowskian gravity, Phys. Rev. D 102 (2020) 024060 [arXiv: 1912.02139] [INSPIRE]. 
[55] Z. Bern, A. Luna, R. Roiban, C.-H. Shen and M. Zeng, Spinning black hole binary dynamics, scattering amplitudes, and effective field theory, Phys. Rev. D 104 (2021) 065014 [arXiv: 2005.03071] [INSPIRE].

[56] Z. Bern, J. Parra-Martinez, R. Roiban, E. Sawyer and C.-H. Shen, Leading nonlinear tidal effects and scattering amplitudes, JHEP 05 (2021) 188 [arXiv:2010.08559] [INSPIRE].

[57] C. Cheung and M.P. Solon, Tidal effects in the post-Minkowskian expansion, Phys. Rev. Lett. 125 (2020) 191601 [arXiv:2006.06665] [INSPIRE].

[58] R. Aoude, K. Haddad and A. Helset, Tidal effects for spinning particles, JHEP 03 (2021) 097 [arXiv: 2012.05256] [INSPIRE].

[59] Z. Bern et al., Scattering amplitudes and conservative binary dynamics at $\mathcal{O}\left(G^{4}\right)$, Phys. Rev. Lett. 126 (2021) 171601 [arXiv:2101.07254] [INSPIRE].

[60] T. Damour, Radiative contribution to classical gravitational scattering at the third order in G, Phys. Rev. D 102 (2020) 124008 [arXiv:2010.01641] [INSPIRE].

[61] P. Di Vecchia, C. Heissenberg, R. Russo and G. Veneziano, Universality of ultra-relativistic gravitational scattering, Phys. Lett. B 811 (2020) 135924 [arXiv:2008.12743] [INSPIRE].

[62] D. Kosmopoulos and A. Luna, Quadratic-in-spin Hamiltonian at $\mathcal{O}\left(G^{2}\right)$ from scattering amplitudes, JHEP 07 (2021) 037 [arXiv:2102.10137] [INSPIRE].

[63] D. Amati, M. Ciafaloni and G. Veneziano, Higher order gravitational deflection and soft bremsstrahlung in Planckian energy superstring collisions, Nucl. Phys. B 347 (1990) 550 [INSPIRE].

[64] P. Di Vecchia, A. Luna, S.G. Naculich, R. Russo, G. Veneziano and C.D. White, A tale of two exponentiations in $N=8$ supergravity, Phys. Lett. B 798 (2019) 134927 [arXiv: 1908. 05603] [INSPIRE].

[65] P. Di Vecchia, S.G. Naculich, R. Russo, G. Veneziano and C.D. White, A tale of two exponentiations in $N=8$ supergravity at subleading level, JHEP 03 (2020) 173 [arXiv: 1911.11716] [INSPIRE].

[66] Z. Bern, H. Ita, J. Parra-Martinez and M.S. Ruf, Universality in the classical limit of massless gravitational scattering, Phys. Rev. Lett. 125 (2020) 031601 [arXiv:2002.02459] [INSPIRE].

[67] M. Accettulli Huber, A. Brandhuber, S. De Angelis and G. Travaglini, From amplitudes to gravitational radiation with cubic interactions and tidal effects, Phys. Rev. D 103 (2021) 045015 [arXiv: 2012.06548] [INSPIRE].

[68] P. Di Vecchia, C. Heissenberg, R. Russo and G. Veneziano, Radiation reaction from soft theorems, Phys. Lett. B 818 (2021) 136379 [arXiv:2101.05772] [INSPIRE].

[69] Y.F. Bautista and A. Guevara, From scattering amplitudes to classical physics: universality, double copy and soft theorems, arXiv:1903.12419 [INSPIRE].

[70] E. Herrmann, J. Parra-Martinez, M.S. Ruf and M. Zeng, Gravitational bremsstrahlung from reverse unitarity, Phys. Rev. Lett. 126 (2021) 201602 [arXiv:2101.07255] [INSPIRE].

[71] E. Herrmann, J. Parra-Martinez, M.S. Ruf and M. Zeng, Radiative classical gravitational observables at $\mathcal{O}\left(G^{3}\right)$ from scattering amplitudes, JHEP 10 (2021) 148 [arXiv:2104.03957] [INSPIRE]. 
[72] P.H. Damgaard, K. Haddad and A. Helset, Heavy black hole effective theory, JHEP 11 (2019) 070 [arXiv: 1908.10308] [INSPIRE].

[73] R. Aoude, K. Haddad and A. Helset, On-shell heavy particle effective theories, JHEP 05 (2020) 051 [arXiv: 2001.09164] [INSPIRE].

[74] A. Brandhuber, G. Chen, G. Travaglini and C. Wen, A new gauge-invariant double copy for heavy-mass effective theory, JHEP 07 (2021) 047 [arXiv:2104.11206] [INSPIRE].

[75] A. Brandhuber, G. Chen, G. Travaglini and C. Wen, Classical gravitational scattering from a gauge-invariant double copy, JHEP 10 (2021) 118 [arXiv:2108.04216] [INSPIRE].

[76] A. Cristofoli, R. Gonzo, D.A. Kosower and D. O'Connell, Waveforms from amplitudes, arXiv:2107.10193 [INSPIRE].

[77] S. Mougiakakos, M.M. Riva and F. Vernizzi, Gravitational bremsstrahlung in the post-Minkowskian effective field theory, Phys. Rev. D 104 (2021) 024041 [arXiv:2102.08339] [INSPIRE].

[78] P.S. Howe, S. Penati, M. Pernici and P.K. Townsend, Wave equations for arbitrary spin from quantization of the extended supersymmetric spinning particle, Phys. Lett. B 215 (1988) 555 [INSPIRE].

[79] F. Bastianelli, P. Benincasa and S. Giombi, Worldline approach to vector and antisymmetric tensor fields, JHEP 04 (2005) 010 [hep-th/0503155] [INSPIRE].

[80] F. Bastianelli, P. Benincasa and S. Giombi, Worldline approach to vector and antisymmetric tensor fields. II, JHEP 10 (2005) 114 [hep-th/0510010] [INSPIRE].

[81] R.A. Porto, The effective field theorist's approach to gravitational dynamics, Phys. Rept. 633 (2016) 1 [arXiv: 1601.04914] [inSPIRE].

[82] M. Levi, Effective field theories of post-Newtonian gravity: a comprehensive review, Rept. Prog. Phys. 83 (2020) 075901 [arXiv: 1807.01699] [InSPIRE].

[83] W.D. Goldberger, J. Li and S.G. Prabhu, Spinning particles, axion radiation, and the classical double copy, Phys. Rev. D 97 (2018) 105018 [arXiv:1712.09250] [InSPIRE].

[84] W.D. Goldberger and A.K. Ridgway, Radiation and the classical double copy for color charges, Phys. Rev. D 95 (2017) 125010 [arXiv:1611.03493] [INSPIRE].

[85] C.-H. Shen, Gravitational radiation from color-kinematics duality, JHEP 11 (2018) 162 [arXiv: 1806. 07388] [INSPIRE].

[86] R.A. Porto, A. Ross and I.Z. Rothstein, Spin induced multipole moments for the gravitational wave flux from binary inspirals to third post-Newtonian order, JCAP 03 (2011) 009 [arXiv: 1007.1312] [INSPIRE].

[87] R.A. Porto, A. Ross and I.Z. Rothstein, Spin induced multipole moments for the gravitational wave amplitude from binary inspirals to 2.5 post-Newtonian order, JCAP 09 (2012) 028 [arXiv: 1203.2962] [INSPIRE].

[88] N.T. Maia, C.R. Galley, A.K. Leibovich and R.A. Porto, Radiation reaction for spinning bodies in effective field theory I: spin-orbit effects, Phys. Rev. D 96 (2017) 084064 [arXiv: 1705. 07934] [INSPIRE].

[89] N.T. Maia, C.R. Galley, A.K. Leibovich and R.A. Porto, Radiation reaction for spinning bodies in effective field theory II: spin-spin effects, Phys. Rev. D 96 (2017) 084065 [arXiv: 1705. 07938] [INSPIRE]. 
[90] G. Cho, B. Pardo and R.A. Porto, Gravitational radiation from inspiralling compact objects: spin-spin effects completed at the next-to-leading post-Newtonian order, Phys. Rev. D 104 (2021) 024037 [arXiv: 2103.14612] [INSPIRE].

[91] C.K. Mishra, A. Kela, K.G. Arun and G. Faye, Ready-to-use post-Newtonian gravitational waveforms for binary black holes with nonprecessing spins: an update, Phys. Rev. D 93 (2016) 084054 [arXiv: 1601.05588] [inSPIRE].

[92] A. Buonanno, G. Faye and T. Hinderer, Spin effects on gravitational waves from inspiraling compact binaries at second post-Newtonian order, Phys. Rev. D 87 (2013) 044009 [arXiv:1209.6349] [INSPIRE].

[93] J. Vines, Scattering of two spinning black holes in post-Minkowskian gravity, to all orders in spin, and effective-one-body mappings, Class. Quant. Grav. 35 (2018) 084002 [arXiv: 1709.06016] [INSPIRE].

[94] D. Bini and T. Damour, Gravitational spin-orbit coupling in binary systems, post-Minkowskian approximation and effective one-body theory, Phys. Rev. D 96 (2017) 104038 [arXiv: 1709.00590] [INSPIRE].

[95] D. Bini and T. Damour, Gravitational spin-orbit coupling in binary systems at the second post-Minkowskian approximation, Phys. Rev. D 98 (2018) 044036 [arXiv:1805.10809] [INSPIRE].

[96] A. Guevara, Holomorphic classical limit for spin effects in gravitational and electromagnetic scattering, JHEP 04 (2019) 033 [arXiv:1706.02314] [INSPIRE].

[97] J. Vines, J. Steinhoff and A. Buonanno, Spinning-black-hole scattering and the test-black-hole limit at second post-Minkowskian order, Phys. Rev. D 99 (2019) 064054 [arXiv: 1812.00956] [INSPIRE].

[98] A. Guevara, A. Ochirov and J. Vines, Scattering of spinning black holes from exponentiated soft factors, JHEP 09 (2019) 056 [arXiv: 1812.06895] [INSPIRE].

[99] M.-Z. Chung, Y.-T. Huang, J.-W. Kim and S. Lee, The simplest massive S-matrix: from minimal coupling to black holes, JHEP 04 (2019) 156 [arXiv:1812.08752] [INSPIRE].

[100] A. Guevara, A. Ochirov and J. Vines, Black-hole scattering with general spin directions from minimal-coupling amplitudes, Phys. Rev. D 100 (2019) 104024 [arXiv:1906.10071] [INSPIRE].

[101] M.-Z. Chung, Y.-T. Huang and J.-W. Kim, Classical potential for general spinning bodies, JHEP 09 (2020) 074 [arXiv: 1908.08463] [INSPIRE].

[102] A. Guevara, B. Maybee, A. Ochirov, D. O'connell and J. Vines, A worldsheet for Kerr, JHEP 03 (2021) 201 [arXiv: 2012.11570] [INSPIRE].

[103] L. Brink, S. Deser, B. Zumino, P. Di Vecchia and P.S. Howe, Local supersymmetry for spinning particles, Phys. Lett. B 64 (1976) 435 [Erratum ibid. 68 (1977) 488] [INSPIRE].

[104] P.S. Howe, S. Penati, M. Pernici and P.K. Townsend, A particle mechanics description of antisymmetric tensor fields, Class. Quant. Grav. 6 (1989) 1125 [INSPIRE].

[105] F. Bastianelli, O. Corradini and A. Zirotti, Dimensional regularization for $N=1$ supersymmetric sigma models and the worldline formalism, Phys. Rev. D 67 (2003) 104009 [hep-th/0211134] [INSPIRE]. 
[106] R. Bonezzi, A. Meyer and I. Sachs, Einstein gravity from the $N=4$ spinning particle, JHEP 10 (2018) 025 [arXiv: 1807.07989] [INSPIRE].

[107] J. Vines, D. Kunst, J. Steinhoff and T. Hinderer, Canonical Hamiltonian for an extended test body in curved spacetime: to quadratic order in spin, Phys. Rev. D 93 (2016) 103008 [Erratum ibid. 104 (2021) 029902] [arXiv:1601.07529] [INSPIRE].

[108] F. Bastianelli and P. van Nieuwenhuizen, Trace anomalies from quantum mechanics, Nucl. Phys. B 389 (1993) 53 [hep-th/9208059] [INSPIRE].

[109] F. Bastianelli, O. Corradini and P. van Nieuwenhuizen, Dimensional regularization of the path integral in curved space on an infinite time interval, Phys. Lett. B 490 (2000) 154 [hep-th/0007105] [INSPIRE].

[110] A. Cristofoli, P.H. Damgaard, P. Di Vecchia and C. Heissenberg, Second-order post-Minkowskian scattering in arbitrary dimensions, JHEP 07 (2020) 122 [arXiv: 2003.10274] [INSPIRE].

[111] A. Koemans Collado, P. Di Vecchia and R. Russo, Revisiting the second post-Minkowskian eikonal and the dynamics of binary black holes, Phys. Rev. D 100 (2019) 066028 [arXiv: 1904.02667] [INSPIRE].

[112] N. Arkani-Hamed, Y.-T. Huang and D. O'Connell, Kerr black holes as elementary particles, JHEP 01 (2020) 046 [arXiv: 1906.10100] [INSPIRE].

[113] C. Shi and J. Plefka, Classical double copy of worldline quantum field theory, arXiv:2109.10345 [INSPIRE].

[114] P. Di Vecchia, C. Heissenberg, R. Russo and G. Veneziano, The eikonal approach to gravitational scattering and radiation at $\mathcal{O}\left(G^{3}\right)$, JHEP 07 (2021) 169 [arXiv:2104.03256] [INSPIRE].

[115] N. Ahmadiniaz, V.M. Banda Guzmán, F. Bastianelli, O. Corradini, J.P. Edwards and C. Schubert, Worldline master formulas for the dressed electron propagator. Part I. Off-shell amplitudes, JHEP 08 (2020) 049 [arXiv: 2004.01391] [INSPIRE].

[116] N. Ahmadiniaz, V.M.B. Guzman, F. Bastianelli, O. Corradini, J.P. Edwards and C. Schubert, Worldline master formulas for the dressed electron propagator, part 2: on-shell amplitudes, arXiv:2107.00199 [INSPIRE].

[117] C. Schubert, Perturbative quantum field theory in the string inspired formalism, Phys. Rept. 355 (2001) 73 [hep-th/0101036] [INSPIRE].

[118] J.P. Edwards and C. Schubert, Quantum mechanical path integrals in the first quantised approach to quantum field theory, arXiv:1912.10004 [INSPIRE].

[119] J.P. Edwards, C.M. Mata, U. Müller and C. Schubert, New techniques for worldline integration, SIGMA 17 (2021) 065 [arXiv:2106.12071] [INSPIRE].

[120] R.N. Lee, Presenting LiteRed: a tool for the Loop InTEgrals REDuction, arXiv:1212.2685 [INSPIRE].

[121] R.N. Lee, LiteRed 1.4: a powerful tool for reduction of multiloop integrals, J. Phys. Conf. Ser. 523 (2014) 012059 [arXiv: 1310.1145] [INSPIRE].

[122] A.V. Smirnov and F.S. Chuharev, FIRE6: Feynman Integral REduction with modular arithmetic, Comput. Phys. Commun. 247 (2020) 106877 [arXiv:1901.07808] [INSPIRE]. 